\title{
Convergence and sharp thresholds for propagation in nonlinear diffusion problems
}

Received July 22, 2008 and in revised form December 3, 2008

Abstract. We study the Cauchy problem

$$
u_{t}=u_{x x}+f(u) \quad\left(t>0, x \in \mathbb{R}^{1}\right), \quad u(0, x)=u_{0}(x) \quad\left(x \in \mathbb{R}^{1}\right),
$$

where $f(u)$ is a locally Lipschitz continuous function satisfying $f(0)=0$. We show that any nonnegative bounded solution with compactly supported initial data converges to a stationary solution as $t \rightarrow \infty$. Moreover, the limit is either a constant or a symmetrically decreasing stationary solution. We also consider the special case where $f$ is a bistable nonlinearity and the case where $f$ is a combustion type nonlinearity. Examining the behavior of a parameter-dependent solution $u_{\lambda}$, we show the existence of a sharp threshold between extinction (i.e., convergence to 0 ) and propagation (i.e., convergence to 1 ). The result holds even if $f$ has a jumping discontinuity at $u=1$.

Keywords. Nonlinear diffusion equation, asymptotic behavior, omega limit set, Cauchy problem, Allen-Cahn, combustion, sharp threshold

\section{Introduction}

We consider the Cauchy problem

$$
\begin{cases}u_{t}=u_{x x}+f(u), & (t, x) \in \mathbb{R}_{+} \times \mathbb{R}^{1}, \\ u(0, x)=u_{0}(x) \geq 0, & x \in \mathbb{R}^{1},\end{cases}
$$

where $\mathbb{R}_{+}=(0, \infty), \mathbb{R}^{1}=(-\infty, \infty)$, and $f:[0, \infty) \rightarrow \mathbb{R}^{1}$ is a locally Lipschitz continuous function satisfying

$$
f(0)=0 .
$$

Later we will also consider the case where $f$ has a discontinuity at $u=1$.

Y. Du: Department of Mathematics, School of Science and Technology, University of New England, Armidale, NSW2351, Australia, and Department of Mathematics, Qufu Normal University, P.R. China; e-mail: ydu@turing.une.edu.au

H. Matano: Graduate School of Mathematical Sciences, University of Tokyo, 3-8-1 Komaba, Tokyo 153-8914, Japan; e-mail: matano@ms.u-tokyo.ac.jp

Mathematics Subject Classification (2010): Primary 35K15, 35K55; Secondary 35B40 
The initial data $u_{0}$ is assumed to be in $L^{\infty}\left(\mathbb{R}^{1}\right)$, but we do not necessarily assume its continuity. Thus the initial condition in 1.1 is understood in the following sense:

$$
\lim _{t \rightarrow 0} u(t, x)=u_{0}(x) \quad \text { for a.e. } x \in \mathbb{R}^{1}, \quad \limsup _{t \rightarrow 0}\|u(t, \cdot)\|_{L^{\infty}\left(\mathbb{R}^{1}\right)}<\infty .
$$

It is well-known that, for each bounded nonnegative initial data $u_{0}$, 1.1 possesses a unique nonnegative local-in-time solution that has the above property. Moreover, the solution is smooth for $t>0$, and is defined as long as it stays finite.

The stationary problem for 1.1 is written as

$$
v^{\prime \prime}+f(v)=0, \quad x \in \mathbb{R}^{1} .
$$

The solutions of (1.3) can be classified into the following categories:

(1) constant solutions; in other words, zeros of $f$;

(2) periodic solutions;

(3) symmetrically decreasing solutions, that is, for some $a \in \mathbb{R}^{1}$,

$$
v(x) \equiv v(2 a-x) \quad \text { and } \quad v^{\prime}(x)<0 \quad \text { for } x \in(a, \infty)
$$

(4) symmetrically increasing solutions, that is, for some $a \in \mathbb{R}^{1}$,

$$
v(x) \equiv v(2 a-x) \quad \text { and } \quad v^{\prime}(x)>0 \quad \text { for } x \in(a, \infty) ;
$$

(5) strictly increasing or decreasing solutions.

Let us introduce some more notations. For each $\phi \in L^{\infty}\left(\mathbb{R}^{1}\right)$, we define its support $\operatorname{spt}(\phi)$ as the smallest closed set $A \subset \mathbb{R}^{1}$ such that $\phi=0$ a.e. in $\mathbb{R}^{1} \backslash A$. We use the notation $[\operatorname{spt}(\phi)]$ to denote the convex hull of $\operatorname{spt}(\phi)$. This is the smallest closed interval containing $\operatorname{spt}(\phi)$.

Our first main result is the following:

Theorem 1.1 (Convergence theorem). Let $f$ satisfy 1.2 and let $u_{0} \in L^{\infty}\left(\mathbb{R}^{1}\right)$ be nonnegative and compactly supported. Suppose that the solution $u(t, x)$ of $(1.1)$ is globally defined for $t \geq 0$ and remains bounded as $t \rightarrow \infty$. Then $u$ converges to a stationary solution as $t \rightarrow \infty$ locally uniformly in $\mathbb{R}^{1}$. Moreover, the limit is either a constant solution or a symmetrically decreasing solution of 1.3 .

Remark 1.2. From the above theorem we see that $u$ never converges to a periodic stationary solution. We also remark that certain types of constant solutions of (1.3) can never be the limit of $u$; see Lemma 3.5 for details.

Note that we assume no sign condition nor differentiability of $f$. The condition 1.2 is all we need for the theorem to hold. A convergence theorem for such a general nonlinearity $f$ has been known for problems on a bounded interval or a circle (see, for example, $\left[\mathrm{Ze},[\mathrm{M}, \mathrm{C1}, \mathrm{CM}]\right.$ ), but it is new for an unbounded spatial domain such as $\mathbb{R}^{1}$. 
Our second main result is concerned with the existence of a "sharp threshold" between extinction and propagation. We consider two types of nonlinearities:

$\left(\mathrm{f}_{B}\right)$ the bistable nonlinearity, $\left(\mathrm{f}_{C}\right)$ the combustion nonlinearity.

In the bistable case $\left(\mathrm{f}_{B}\right)$, we assume that $f$ is locally Lipschitz and satisfies

$$
f(0)=f\left(\theta_{0}\right)=f(1)=0, \quad f(s) \begin{cases}<0 & \text { in }\left(0, \theta_{0}\right) \cup(1, \infty), \\ >0 & \text { in }\left(\theta_{0}, 1\right),\end{cases}
$$

for some $\theta_{0} \in(0,1)$ and

$$
\int_{0}^{1} f(s) d s>0
$$

Such a nonlinearity appears in various applications including mathematical ecology, population genetics and physics. See, for example, [F, NAY, AC, AW1, AW2, FM] and the references therein.

In the combustion case $\left(\mathrm{f}_{C}\right)$, we assume that $f$ is locally Lipschitz and satisfies

$$
f(s)=0 \quad \text { in }\left[0, \theta_{0}\right], \quad f(s)>0 \quad \text { in }\left(\theta_{0}, 1\right), \quad f(s) \leq 0 \quad \text { in }[1, \infty)
$$

for some $\theta_{0} \in(0,1)$, and that there exists $\delta_{0}>0$ such that

$$
f(s) \text { is nondecreasing in }\left(\theta_{0}, \theta_{0}+\delta_{0}\right) .
$$

(The case $\theta_{0}=0$ will be discussed in Remark 4.12,) Such a nonlinearity appears, typically, as a model for combustion; see $[\overline{\mathrm{ZFK}}, \underline{\mathrm{K}}, \overline{\mathrm{BN}}, \mathrm{Z}]$ and the references therein. The value $\theta_{0}$ is called the "ignition temperature". (The case $\theta_{0}=0$ was first investigated by Fisher $[\mathrm{F}]$ and Kolmogorov-Petrovskiü-Piskunov [KPP], and is known as Fisher's equation or the KPP equation, which was used to describe the propagation of advantageous genes in a population.)

Both in case $\left(\mathrm{f}_{B}\right)$ and in case $\left(\mathrm{f}_{C}\right)$, the following typical behaviors are known to occur:

$$
\text { Extinction: } \quad \lim _{t \rightarrow \infty} u(t, x)=0 ; \quad \text { Propagation: } \quad \lim _{t \rightarrow \infty} u(t, x)=1 .
$$

Our goal in the second half of the paper is to show that there is a sharp transition between extinction and propagation when we vary the initial data.

To put the sharp transition phenomenon into perspective, let us look at the set of bounded nonnegative solutions of $(1.3)$ with $f$ of type $\left(\mathrm{f}_{B}\right)$ or $\left(\mathrm{f}_{C}\right)$. First, in the case of the bistable nonlinearity $\left(f_{B}\right)$, it is easy to see that the bounded nonnegative solutions of (1.3) consist of the following:

$\left(1_{B}\right)$ constant solutions: $0, \theta_{0}, 1$;

$\left(2_{B}\right)$ a family of periodic solutions satisfying $0<\min v<\theta_{0}<\max v<\theta^{*}$;

$\left(3_{B}\right)$ symmetrically decreasing solutions $U(\cdot-a), a \in \mathbb{R}^{1}$, where

$$
U^{\prime \prime}+f(U)=0 \quad \text { in } \mathbb{R}^{1}, \quad U(0)=\theta^{*}, \quad U^{\prime}(0)=0,
$$

which necessarily satisfies $\lim _{|x| \rightarrow \infty} U(x)=0$. 
Here $\theta^{*} \in\left(\theta_{0}, 1\right)$ is the constant determined by the condition

$$
\int_{0}^{\theta^{*}} f(s) d s=0 .
$$

In order to describe the threshold in the sharp transition, we introduce a one-parameter family of initial data $u_{0}=\phi_{\lambda}(\lambda>0)$ satisfying the following conditions, where $X^{+}:=$ $\left\{\phi \in L^{\infty}\left(\mathbb{R}^{1}\right): \phi \geq 0, \operatorname{spt}(\phi)\right.$ is compact $\}$ :

$\left(\Phi_{1}\right) \phi_{\lambda} \in X^{+}$for every $\lambda>0$, and the map $\lambda \mapsto \phi_{\lambda}$ is continuous from $\mathbb{R}_{+}$to $L^{1}\left(\mathbb{R}^{1}\right)$; $\left(\Phi_{2}\right)$ if $0<\lambda_{1}<\lambda_{2}$, then $\phi_{\lambda_{1}} \leq \phi_{\lambda_{2}}$ and $\phi_{\lambda_{1}} \not \equiv \phi_{\lambda_{2}}$ in the a.e. sense;

$\left(\Phi_{3}\right) \lim _{\lambda \rightarrow 0} \phi_{\lambda}(x)=0$ a.e. in $\mathbb{R}^{1}$.

Theorem 1.3 (Threshold for the bistable case). Let $f$ be a locally Lipschitz continuous function satisfying (1.4), (1.5) and let $\left\{\phi_{\lambda}\right\}_{\lambda>0}$ satisfy $\left(\Phi_{1}\right)$ through $\left(\Phi_{3}\right)$. Denote by $u_{\lambda}(t, x)$ the solution of (1.1) with initial data $u_{0}=\phi_{\lambda}$. Then one of the following holds:

(a) $\lim _{t \rightarrow \infty} u_{\lambda}(t, x)=0$ uniformly in $\mathbb{R}^{1}$ for every $\lambda>0$;

(b) there exist $\lambda^{*}>0$ and $x_{0} \in \mathbb{R}^{1}$ such that

$$
\lim _{t \rightarrow \infty} u_{\lambda}(t, x)=\left\{\begin{array}{lll}
0 & \text { uniformly in } \mathbb{R}^{1} & \left(0<\lambda<\lambda^{*}\right), \\
U\left(x-x_{0}\right) & \text { uniformly in } \mathbb{R}^{1} & \left(\lambda=\lambda^{*}\right), \\
1 & \text { locally uniformly in } \mathbb{R}^{1} & \left(\lambda>\lambda^{*}\right)
\end{array}\right.
$$

Next we consider the case of combustion nonlinearity $\left(f_{C}\right)$. In this case, bounded nonnegative solutions of 1.3 are the following constants:

$$
v(x) \equiv \theta \in\left[0, \theta_{0}\right] \cup\{1\} \cup S_{1},
$$

where $S_{1}$ denotes the set of all zeros of $f$ that are larger than 1 (possibly empty). The corresponding threshold theorem is stated as follows:

Theorem 1.4 (Threshold for the combustion case). Let $f$ be a locally Lipschitz continuous function satisfying (1.6), (1.7) and let $\left\{\phi_{\lambda}\right\}_{\lambda>0}$ satisfy $\left(\Phi_{1}\right)$ through $\left(\Phi_{3}\right)$. Denote by $u_{\lambda}(t, x)$ the solution of (1.1) with initial data $u_{0}=\phi_{\lambda}$. Then one of the following holds:

(a) $\lim _{t \rightarrow \infty} u_{\lambda}(t, x)=0$ uniformly in $\mathbb{R}^{1}$ for every $\lambda>0$;

(b) there exists $\lambda^{*}>0$ such that

$$
\lim _{t \rightarrow \infty} u_{\lambda}(t, x)=\left\{\begin{array}{lll}
0 & \text { uniformly in } \mathbb{R}^{1} & \left(0<\lambda<\lambda^{*}\right), \\
\theta_{0} & \text { locally uniformly in } \mathbb{R}^{1} & \left(\lambda=\lambda^{*}\right), \\
1 & \text { locally uniformly in } \mathbb{R}^{1} & \left(\lambda>\lambda^{*}\right) .
\end{array}\right.
$$

Remark 1.5. The conclusions of Theorems 1.3 and 1.4 remain valid even if $f(u)$ has a discontinuous jump at $u=1$. This answers a question raised by Brezis; see Section 6 for details.

Let us give two typical examples of $\phi_{\lambda}$ satisfying the conditions $\left(\Phi_{1}\right)$ through $\left(\Phi_{3}\right)$ : 
Example 1.6. If $\phi \in L^{\infty}\left(\mathbb{R}^{1}\right)$ is an arbitrary nonnegative function with nonempty compact support, then

$$
\phi_{\lambda}(x)=\lambda \phi(x)
$$

clearly satisfies $\left(\Phi_{1}\right)$ to $\left(\Phi_{3}\right)$. Furthermore, the alternative (b) of Theorems 1.3 and 1.4 always holds if $f$ is globally Lipschitz. In fact, under this assumption on $f$, we can show

$$
u_{\lambda}(t, x) \geq \lambda e^{-M t} w(t, x),
$$

where $w$ is the solution of $w_{t}=w_{x x}$ with initial data $\phi$ and $M$ is the Lipschitz coefficient of $f$. To use the above inequality, we simply fix any $t>0$, choose $\lambda$ sufficiently large and then apply Lemmas 4.2 and 4.8 .

Example 1.7. Given arbitrary points $x_{1}<\cdots<x_{k}$ and constants $\sigma_{i}>0(i=1, \ldots, k)$, we set

$$
\phi_{\lambda}(x)=\sum_{i=1}^{k} \sigma_{i} \chi_{\left[x_{i}-\lambda, x_{i}+\lambda\right]}(x),
$$

where $\chi_{A}$ denotes the characteristic function of a set $A \subset \mathbb{R}^{1}$. Then $\left(\Phi_{1}\right)$ to $\left(\Phi_{3}\right)$ are clearly satisfied. Moreover, by Lemmas 4.2 and 4.8, the alternative (b) occurs if $\sum_{i=1}^{k} \sigma_{i}>\theta_{0}$. On the other hand, if $\sum_{i=1}^{k} \sigma_{i}<\theta_{0}$, one can easily check that alternative (a) occurs.

The present work was motivated by a recent paper of Zlatoš [Z], where the conclusions of Theorems 1.3 and 1.4 were shown to hold for the particular family of initial data $u_{0}(x)=\chi_{[-\lambda, \lambda]}(x), \lambda \in(0, \infty)$. The results of [Z] gave a positive answer to a question left open in the work of Kanel [K] and Aronson-Weinberger [AW1]. In [K], it was shown that in the combustion case with $u_{0}(x)=\chi_{[-\lambda, \lambda]}(x)$, there exists $0<\lambda_{0} \leq \lambda_{1}$ such that extinction occurs for $\lambda<\lambda_{0}$, and propagation occurs for $\lambda>\lambda_{1}$. The same was shown to hold for the bistable case in [AW1]. However, the question of whether $\lambda_{0}=\lambda_{1}$ or not remained open until [Z]. While the methods used in [Z] can be extended to treat more general initial data $u_{0}$ than the characteristic function of an interval, the arguments there depend crucially on the symmetry and monotonicity properties of $u_{0}(x)$. Our theorems, on the other hand, are applicable to a much wider class of initial data.

The proof of Theorem 1.1 is based on a zero number argument, the reflection method and estimates near $|x|=\infty$. In the special case of bistable nonlinearity $\left(\mathrm{f}_{B}\right)$, one can also use a Lyapunov functional (the energy) to partly simplify the argument (see Remark 4.6. However, unlike the problems in a bounded domain, this approach does not work under the general assumption [1.2], since the energy may tend to $-\infty$ as $t \rightarrow \infty$ for most solutions. For this reason we have avoided the use of the energy and based our argument on the zero number properties.

To be more precise, the proof of Theorem 1.1 consists of two steps. First we show that any element of $\omega(u)$ is a solution of (1.3). Here $\omega(u)$ denotes the $\omega$-limit set of the solution $u$. Secondly, we show that $\omega(u)$ contains only one element. A key observation for establishing the first claim is that, for any $w \in \omega(u)$ and for any solution $v$ of 1.3 . which is positive in $\mathbb{R}^{1}$ or has compact positive support, either $w(x)-v(x)$ has only 
simple zeros, or $w(x) \equiv v(x)$. This can be shown by studying the number of zeros of $u(t, x)-v(x)$ as $t$ approaches $\infty$. The second claim is established partly by showing that there exists $x_{0} \in \mathbb{R}^{1}$ such that

$$
w^{\prime}\left(x_{0}\right)=0 \quad \text { for any } w \in \omega(u) .
$$

This is done by using the reflection argument found in [C1, CM].

The key point in Theorems 1.3 and 1.4 is the assertion that the situation $u_{\lambda} \nrightarrow 0,1$ $(t \rightarrow \infty)$ occurs only for a single value of $\lambda=\lambda^{*}$. The rest follows easily from Theorem 1.1 and the comparison principle. The proof of this sharp threshold result differs between the case of bistable nonlinearity $\left(\mathrm{f}_{B}\right)$ and the case of combustion nonlinearity $\left(\mathrm{f}_{C}\right)$, but in either case a key step is established by making use of the following proposition. Since this proposition holds in any space dimension, we state it in a rather general framework:

Propostion 1.8. Suppose that $g:[0, \infty) \rightarrow \mathbb{R}^{1}$ is locally Lipschitz continuous, $g(0)=0$, and that $u_{0} \in L^{\infty}\left(\mathbb{R}^{N}\right)$ is nonnegative and has nonempty compact support $\operatorname{spt}\left(u_{0}\right)$. Then there exist $\delta, R>0$ (depending on $g$ and $u_{0}$ ) such that the solution of the Cauchy problem

$$
\begin{cases}u_{t}=\Delta u+g(u), & (t, x) \in \mathbb{R}_{+} \times \mathbb{R}^{N}, \\ u(0, x)=u_{0}(x), & x \in \mathbb{R}^{N},\end{cases}
$$

satisfies $u_{t}(t, x)>0$ for all $t \in(0, \delta]$ and $|x| \geq R$.

The above proposition follows easily from the following identity, which we will prove in Section 5

$$
\lim _{t \downarrow 0,|x| \rightarrow \infty}\left(\frac{t}{|x|}\right)^{2} \frac{u_{t}(t, x)}{u(t, x)}=\frac{1}{4} .
$$

We now give a brief overview of related research. The front propagation in (1.1) for the cases $\left(\mathrm{f}_{B}\right)$ and $\left(\mathrm{f}_{C}\right)$ has been studied extensively in the past few decades, and various sufficient conditions on the initial data $u_{0}$ for 'extinction' $\left(\lim _{t \rightarrow \infty} u(t, x)=0\right)$ and for 'propagation' $\left(\lim _{t \rightarrow \infty} u(t, x)=1\right)$ have been found. For example, it is easy to show that if $0 \leq u_{0}(x)<\theta_{0}\left(x \in \mathbb{R}^{N}\right)$, then $u(t, x) \rightarrow 0$ as $t \rightarrow \infty$. In [AW1], it is shown that there exists a continuous function $\phi_{0}$ with compact support such that if $u_{0} \geq \phi_{0}$, then $u(t, x) \rightarrow 1$ as $t \rightarrow \infty$.

If $f$ is $C^{1}$ and $f^{\prime}(0)<0, f^{\prime}(1)<0$, then much more is known. For example, [FM] shows that, for each $\theta>\theta_{0}$, there exists $L=L(\theta)>0$ such that if $u_{0}(x) \geq \theta$ on some interval $I$ with length $|I| \geq L$, then $u(t, x) \rightarrow 1$ as $t \rightarrow \infty$. (See Lemma 4.2 below for a slight extension of this result.) Moreover, the existence, uniqueness and stability of traveling fronts connecting 0 and 1 are proved in [FM]. Under the same assumption on $f$, [J] has studied radially symmetric solutions of a higher dimensional problem

$$
u_{t}=\Delta u+f(u), \quad(t, x) \in \mathbb{R}_{+} \times \mathbb{R}^{N},
$$

and obtained sharp estimates on the speed of propagation. 
More recently, under the assumption that $f \in C^{1}, f(0)=0, f^{\prime}(0)<0$, [BJP] has proved the convergence of bounded solutions of 1.13 under the condition that $u(t, x)$ decays to 0 as $|x| \rightarrow \infty$ uniformly in $t>0$. This last condition is automatically fulfilled if $f$ is a bistable nonlinearity ( $\left.\mathrm{f}_{B}\right)$ (plus $f \in C^{1}$ and $f^{\prime}(0)<0$ ), if $u_{0}(x)$ is nonnegative and decays to 0 exponentially fast as $|x| \rightarrow \infty$, and if $u \nrightarrow 1$ as $t \rightarrow \infty$. Their approach makes substantial use of the condition $f^{\prime}(0)<0$ and relies on a dynamical system method based on the properties of invariant manifolds in a neighborhood of equilibria, which requires $f \in C^{1}$.

Our approach is very different and need not assume such conditions as

$$
f(u) \leq-\sigma u \quad \text { for some } \sigma>0 \text { and all small } u>0,
$$

which is commonly used in the literature including [FM, J, $\mathrm{BJP}]$.

In $[\overline{\mathrm{FP}}]$, the convergence problem for the periodic counterpart of 1.1 was studied, where $f=f(t, u)$ is $C^{1}$ and $\tau$-periodic in $t$ for some $\tau>0$, and satisfies

$$
f(t, 0)=0, \quad f_{u}(t, 0)<0 .
$$

However, they only consider solutions satisfying the following conditions:

(i) $u(t, \cdot) \in C_{0}\left(\mathbb{R}^{1}\right):=\left\{\phi \in C\left(\mathbb{R}^{1}\right): \lim _{|x| \rightarrow \infty} \phi(x)=0\right\}$ for all $t>0$,

(ii) $\left\{u(t, \cdot): t \in \mathbb{R}_{+}\right\}$is relatively compact in $C_{0}\left(\mathbb{R}^{1}\right)$, namely, for any $t_{n}>0,\left\{u\left(t_{n}, \cdot\right)\right\}$ has a subsequence that converges to some $u_{\infty} \in C_{0}\left(\mathbb{R}^{1}\right)$ uniformly in $\mathbb{R}^{1}$.

These restrictions (especially (ii)) force the $\omega$-limit set of a given solution to be an element of a set consisting of (up to translation in $x$ ) at most three elements $0, u_{+}$and $u_{-}$, where $u_{+}$(when it exists) is the unique positive periodic solution with property (i), and $u_{-}$ (when it exists) is the unique negative periodic solution with property (i). The approach in $[\mathrm{FP}]$ used zero number arguments as well as dynamical system arguments based on properties of invariant manifolds in a neighborhood of equilibria, which requires $f \in C^{1}$. In contrast, though our convergence theorem (Theorem 1.1) deals with the autonomous problem 1.1, it does not have such implicit restrictions on the $\omega$-limit set, and it applies to more general $f$.

The paper is organized as follows. In Sections 2 and 3 we prove Theorem 1.1 . In Section 4, we consider parameterized initial data and prove Theorems 1.3 and 1.4 , assuming the validity of Proposition 1.8, whose proof is given in Section 5 . Finally, in Section 6, we extend Theorems 1.1, 1.3 and 1.4 to cover certain important cases with a discontinuous nonlinearity.

\section{Preliminaries}

In this section, we collect some basic facts which will be needed in our proof of Theorem 1.1 in the next section. 


\subsection{Behavior near $|x|=\infty$}

Lemma 2.1. Let $u(t, x)$ be a solution of (1.1) satisfying the assumptions of Theorem 1.1 and put $\left[\operatorname{spt}\left(u_{0}\right)\right]=\left[L_{1}, L_{2}\right]$. Then

$$
u_{x}>0 \text { for } x<L_{1}, t>0 ; \quad u_{x}<0 \text { for } x>L_{2}, t>0 .
$$

Consequently,

$$
\|u(t, \cdot)\|_{L^{\infty}\left(\mathbb{R}^{1}\right)}=\|u(t, \cdot)\|_{L^{\infty}\left(\left(L_{1}, L_{2}\right)\right)} .
$$

Proof. Fix any $x_{0} \in\left(-\infty, L_{1}\right)$ and define $w(t, x)=u(t, x)-u\left(t, 2 x_{0}-x\right)$. Then, since $u$ is bounded and $f$ is locally Lipschtiz continuous, we can write

$$
f(u(t, x))-f\left(u\left(t, 2 x_{0}-x\right)\right)=c(t, x) w(t, x)
$$

with some bounded function $c(t, x)$. Thus $w$ satisfies

$$
\begin{cases}w_{t}=w_{x x}+c(t, x) w & \left(t>0,-\infty<x<x_{0}\right) \\ w\left(t, x_{0}\right)=0 & (t>0) \\ w(0, x) \leq 0 & \left(-\infty<x<x_{0}\right)\end{cases}
$$

and $w(0, x) \not \equiv 0$. Therefore the strong maximum principle yields $w(t, x)<0$ for $t>0$ and $x<x_{0}$. It then follows from the Hopf boundary lemma that $w_{x}\left(t, x_{0}\right)>0$, that is, $2 u_{x}\left(t, x_{0}\right)>0$ for all $t>0$. The case $x_{0}>L_{2}$ can be argued analogously. The lemma is proved.

Lemma 2.2. Any solution of (1.1) as given in Theorem 1.1 satisfies

$$
\lim _{|x| \rightarrow \infty} u(t, x)=0 \quad \text { uniformly in } t \in[0, T] \text { for each } T>0 .
$$

Proof. Since $f$ is locally Lipschitz continuous and $f(0)=0$, and $u(t, x)$ is bounded, there exists a constant $M \geq 0$ such that $f(u(t, x)) \leq M u(t, x)$; hence by the comparison principle, we have

$$
u(t, x) \leq e^{M t} \bar{u}(t, x),
$$

where $\bar{u}$ is the solution of $\bar{u}_{t}=\bar{u}_{x x}$ with initial data $\bar{u}(0, x)=u_{0}(x)$. Since $\bar{u}(t, x) \rightarrow 0$ as $|x| \rightarrow \infty$, we obtain 2.3.

\subsection{Zero number properties}

In what follows, $\mathcal{Z}(w)$ will denote the number of sign changes of a continuous function $w(x)$ defined on $\mathbb{R}^{1}$. Note that, if the zeros of $w$ are all simple, then $\mathcal{Z}(w)$ coincides with the number of zeros of $w$. We also use the notation $\mathcal{Z}_{I}(w)$ to denote the number of sign changes of $w$ on a given interval $I$.

The following well-known lemma is a refined version of the Sturmian theorem for one-dimensional parabolic equations. 
Lemma 2.3. Let $w(t, x) \not \equiv 0$ be a solution of the equation

$$
w_{t}=w_{x x}+c(t, x) w \quad\left(t \in\left(t_{1}, t_{2}\right), x \in \mathbb{R}^{1}\right),
$$

where the coefficient function $c$ is bounded. Then, for each $t \in\left(t_{1}, t_{2}\right)$, the zeros of the function $x \mapsto w(t, x)$ do not accumulate in $\mathbb{R}^{1}$. Furthermore,

(i) $\mathcal{Z}(w(t, \cdot))$ is nonincreasing in $t$;

(ii) if $w\left(t^{*}, x^{*}\right)=w_{x}\left(t^{*}, x^{*}\right)=0$ for some $t^{*} \in\left(t_{1}, t_{2}\right), x^{*} \in \mathbb{R}^{1}$, then

$$
\mathcal{Z}(w(t, \cdot))>\mathcal{Z}(w(s, \cdot)) \quad \text { for all } t \in\left(t_{1}, t^{*}\right), s \in\left(t^{*}, t_{2}\right)
$$

whenever $\mathcal{Z}(w(s, \cdot))<\infty$.

The same assertions hold for $\mathcal{Z}_{I}(w(t, \cdot))$ for any interval $I \subset \mathbb{R}^{1}$, provided that either $w$ never vanishes on the boundary of $I$ or $w \equiv 0$ on the boundary of $I$.

Most of the conclusions in Lemma 2.3 are contained in or are easy consequences of [An], but statement (i) does not follow directly from [An] when $I$ is an infinite interval. However, it can be easily shown by combining the argument in $[\mathrm{M}]$ and the PhragménLindelöf type maximum principle.

Corollary 2.4. Let $w$ be a solution of 2.5]. If $w(t, x)$ has a constant sign near $x=-\infty$ and near $x=\infty$, then

$$
\mathcal{Z}(w(t, \cdot))<\infty
$$

if $\mathcal{Z}\left(w\left(t_{0}, \cdot\right)\right)<\infty$ then

$$
\mathcal{Z}(w(t, \cdot))<\infty \quad \text { for all } t>t_{0}
$$

Corollary 2.5. Let $w$ be a solution of 2.5) and suppose that there exists $x_{0} \in \mathbb{R}^{1}$ such that $w\left(t, x_{0}\right)=w_{x}\left(t, x_{0}\right)=0$ for every $t$ in some interval $t_{1}<t<t_{2}$. Then $w \equiv 0$.

Let us recall other basic properties of $\mathcal{Z}$. Since $\mathcal{Z}$ denotes the number of sign changes, the pointwise convergence $w_{n}(x) \rightarrow w(x)$ implies

$$
w \equiv 0 \quad \text { or } \quad \mathcal{Z}(w) \leq \liminf _{n \rightarrow \infty} \mathcal{Z}\left(w_{n}\right)
$$

Moreover, if $I$ is a compact interval, if $w_{n} \rightarrow w$ in $C^{1}(I)$, if every zero of $w$ in $I$ is simple, and finally if $w \neq 0$ on the boundary of $I$, then

$$
\mathcal{Z}_{I}(w)=\lim _{n \rightarrow \infty} \mathcal{Z}_{I}\left(w_{n}\right)
$$

Lemma 2.6. Let $w_{n}(t, x)$ be a sequence of functions converging to $w(t, x)$ in $C^{1}\left(\left(t_{1}, t_{2}\right) \times I\right)$, where $I$ is an open interval in $\mathbb{R}^{1}$. Assume that, for every $t \in\left(t_{1}, t_{2}\right)$ and $n \in \mathbb{N}$, the function $x \mapsto w_{n}(t, x)$ has only simple zeros in $I$, and that $w(t, x)$ satisfies an equation of the form 2.5] on $\left(t_{1}, t_{2}\right) \times I$. Then for every $t \in\left(t_{1}, t_{2}\right)$, either $w(t, x) \equiv 0$ on $I$, or $w(t, x)$ has only simple zeros in $I$. 
Here and in what follows, we use the usual convention that $u_{n}$ converges to $u$ in $C^{m}(\Omega)$ if and only if $u_{n} \rightarrow u$ in $C^{m}(K)$ for any compact subset $K$ of $\Omega$.

Proof. Suppose that there exists $t^{*} \in\left(t_{1}, t_{2}\right)$ such that $w\left(t^{*}, x\right)$ has a degenerate zero, say $x^{*}$, in $I$ and that $w\left(t^{*}, x\right) \neq \equiv$. By Lemma 2.3, the zeros of $w\left(t^{*}, x\right)$ are isolated, therefore we can choose $\tau, \sigma>0$ sufficiently small so that $x^{*}$ is the only zero of $w\left(t^{*}, x\right)$ in the interval $J:=\left\{\left|x-x^{*}\right| \leq \sigma\right\} \subset I$ and that

$$
w\left(t, x^{*} \pm \sigma\right) \neq 0 \quad \text { for } t \in\left[t^{*}-\tau, t^{*}+\tau\right] .
$$

Since $x^{*}$ is a degenerate zero, by Lemma 2.3 we have

$$
\mathcal{Z}_{J}\left(w\left(t^{*}-\tau, \cdot\right)\right)>\mathcal{Z}_{J}\left(w\left(t^{*}+\tau, \cdot\right)\right) .
$$

Here we can assume without loss of generality that the zeros of $w\left(t^{*} \pm \tau, x\right)$ in the interval $J$ are all simple, since degenerate zeros appear at discrete time moments by Lemma 2.3 (ii). In view of this and (2.7), we see that

$$
\begin{gathered}
\mathcal{Z}_{J}\left(w_{n}\left(t^{*}-\tau, \cdot\right)\right)>\mathcal{Z}_{J}\left(w_{n}\left(t^{*}+\tau, \cdot\right)\right), \\
w_{n}\left(t, x^{*} \pm \sigma\right) \neq 0 \quad \text { for } t \in\left[t^{*}-\tau, t^{*}+\tau\right]
\end{gathered}
$$

for all large $n$, which implies that $w_{n}(t, x)$ has a degenerate zero in the region $(t, x) \in$ $\left(t^{*}-\tau, t^{*}+\tau\right) \times J$, contradicting the assumption of the lemma. This contradiction proves the lemma.

\subsection{Reflection of the solution}

Lemma 2.7. Let $u$ be as in Lemma 2.1 and, for each $a \in \mathbb{R}^{1}$, define

$$
w_{a}(t, x):=u(t, x)-u(t, 2 a-x) .
$$

Then for every $a \neq\left(L_{1}+L_{2}\right) / 2$ and $t>0$, the function $x \mapsto w_{a}(t, x)$ changes sign at most finitely many times.

Proof. Let us write $w(t, x)=w_{a}(t, x)$ for simplicity. Since both $u(t, x)$ and $u(t, 2 a-x)$ satisfy the same equation (1.1), $w$ satisfies a linear parabolic equation of the form

$$
w_{t}=w_{x x}+c(t, x) w
$$

where $c$ is bounded because of the local Lipschitz continuity of $f$.

Denote by $\bar{u}(t, x)$ the solution of the following Cauchy problem:

$$
\bar{u}_{t}=\bar{u}_{x x} \quad\left(t>0, x \in \mathbb{R}^{1}\right), \quad \bar{u}(0, x)=u_{0}(x) \quad\left(x \in \mathbb{R}^{1}\right) .
$$

Since $u$ is bounded and $f$ is locally Lipschitz, $-M u \leq f(u) \leq M u$ for some constant $M \geq 0$ and all $t \geq 0, x \in \mathbb{R}^{1}$. Therefore a simple comparison argument shows that

$$
e^{-M t} \bar{u}(t, x) \leq u(t, x) \leq e^{M t} \bar{u}(t, x) \quad \text { for all }(t, x) \in[0, \infty) \times \mathbb{R}^{1} .
$$


Thus we have

$$
\left\{\begin{array}{l}
w(t, x) \geq e^{-M t} \bar{u}(t, x)-e^{M t} \bar{u}(t, 2 a-x), \\
w(t, x) \leq e^{M t} \bar{u}(t, x)-e^{-M t} \bar{u}(t, 2 a-x)
\end{array}\right.
$$

Note also that

$$
\begin{aligned}
& \bar{u}(t, x)=(4 \pi t)^{-1 / 2} \int_{L_{1}}^{L_{2}} \exp \left(-\frac{(x-y)^{2}}{4 t}\right) u_{0}(y) d y \\
& \bar{u}(t, 2 a-x)=(4 \pi t)^{-1 / 2} \int_{2 a-L_{2}}^{2 a-L_{1}} \exp \left(-\frac{(x-y)^{2}}{4 t}\right) u_{0}(2 a-y) d y .
\end{aligned}
$$

In what follows we consider only the case $a<\left(L_{1}+L_{2}\right) / 2$, since the other case can be treated the same way. In this case, we claim that

$$
\lim _{x \rightarrow-\infty} \frac{\bar{u}(t, 2 a-x)}{\bar{u}(t, x)}=\infty, \quad \lim _{x \rightarrow \infty} \frac{\bar{u}(t, 2 a-x)}{\bar{u}(t, x)}=0 .
$$

To see this, we use 2.9. Choose $L_{*}$ with $2 a-L_{2}<L_{*}<L_{1}$. Then for some constants $C_{1}, C_{2}>0$ we have

$$
\begin{aligned}
\bar{u}(t, x) & \leq C_{1} \exp \left(-\frac{\left(x-L_{1}\right)^{2}}{4 t}\right) \quad \text { for } x \leq L_{1}, \\
\bar{u}(t, 2 a-x) & \geq(4 \pi t)^{-1 / 2} \int_{2 a-L_{2}}^{L_{*}} \exp \left(-\frac{(x-y)^{2}}{4 t}\right) u_{0}(2 a-y) d y \\
& \geq C_{2} \exp \left(-\frac{\left(x-L_{*}\right)^{2}}{4 t}\right) \quad \text { for } x \leq 2 a-L_{2},
\end{aligned}
$$

from which the first assertion of 2.10 follows. The second assertion of 2.10 can be shown similarly. Consequently,

$$
\begin{cases}e^{M t} \bar{u}(t, x)-e^{-M t} \bar{u}(t, 2 a-x)<0 & \text { for } x \ll-1, \\ e^{-M t} \bar{u}(t, x)-e^{M t} \bar{u}(t, 2 a-x)>0 & \text { for } x \gg 1\end{cases}
$$

This and 2.8) imply that, for each $t_{0}>0$, there exists $\ell>0$ such that

$$
w\left(t_{0}, x\right)<0 \text { for } x \leq-\ell, \quad w\left(t_{0}, x\right)>0 \text { for } x \geq \ell .
$$

Thus by Corollary 2.4,

$$
\mathcal{Z}(w(t, \cdot))<\infty \quad \text { for all } t>t_{0} .
$$

Since $t_{0}>0$ is arbitrary, the conclusion of the lemma follows. 


\subsection{Position of peaks}

By Lemma2.1, the positions of the local maxima and minima of the function $x \mapsto u(t, x)$ are confined to the interval $\left[L_{1}, L_{2}\right]$ for every $t>0$. The following lemma implies, roughly speaking, that the positions of the local maxima and minima do not oscillate indefinitely.

Lemma 2.8. For every $x \neq\left(L_{1}+L_{2}\right) / 2$, the following limit exists:

$$
\lim _{t \rightarrow \infty} \operatorname{sgn}\left(u_{x}(t, x)\right) \neq 0,
$$

where $\operatorname{sgn}(w):=1,-1,0$ depending on whether $w>0, w<0$ or $w=0$.

Proof. We use an argument introduced in [C1, CM]. Fix $a \neq\left(L_{1}+L_{2}\right) / 2$ arbitrarily and put $w(t, x):=u(t, x)-u(t, 2 a-x)$. Then by Lemmas 2.7 and 2.3 , $\mathcal{Z}(w(t, \cdot))$ is finite for every $t>0$ and it is nonincreasing in $t$. Therefore $\mathcal{Z}(w(t, \cdot))$ is constant for all large $t$. This implies, by Lemma 2.3 (ii), that the function $x \mapsto w(t, x)$ has only simple zeros in $\mathbb{R}^{1}$ for all large $t$. In particular, since $w(t, a)=0$ for all $t>0$, we have

$$
w_{x}(t, a) \neq 0 \quad \text { for all large } t>0 .
$$

In view of this and the fact that $w_{x}(t, a)$ is continuous in $t$, we see that $w_{x}(t, a)$ does not change sign for all large $t>0$. The conclusion of the lemma now follows since $w_{x}(t, a)=2 u_{x}(t, a)$.

Now we define $x_{0}$ as follows. (Roughly speaking, $x_{0}$ denotes the limit position of the leftmost local maximum of the function $x \mapsto u(t, x)$.)

$$
x_{0}:=\inf \left\{x \in \mathbb{R}^{1}: \lim _{t \rightarrow \infty} \operatorname{sgn}\left(u_{x}(t, x)\right)=-1\right\} .
$$

It is clear from 2.1] that

$$
L_{1} \leq x_{0} \leq L_{2}
$$

\subsection{Intersection with a stationary solution}

If we solve the ordinary differential equation $(1.3)$ under the initial condition

$$
v\left(x_{0}\right)=\alpha, \quad v^{\prime}\left(x_{0}\right)=0
$$

for some $\alpha>0$ and $x_{0} \in \mathbb{R}^{1}$, then $v(x)$ is symmetric about $x=x_{0}$ and either $v(x)>0$ for all $x \in \mathbb{R}^{1}$ or there exists $R>0$ such that

$$
v(x)>0 \quad \text { for }\left|x-x_{0}\right|<R, \quad v\left(x_{0} \pm R\right)=0 .
$$

In the latter case we call $v$ a solution of $(1.3)$ with compact positive support, and define $\operatorname{spt}^{+}(v):=\left[x_{0}-R, x_{0}+R\right]$. In the former case, we define $\operatorname{spt}^{+}(v):=\mathbb{R}^{1}$. 
Lemma 2.9. Let $u$ be as in Lemma 2.1. Suppose that $v$ is either a positive solution of (1.3) in $\mathbb{R}^{1}$ or a solution of (1.3) with compact positive support. Then

$$
\mathcal{Z}_{I}(u(t, \cdot)-v)<\infty \quad \text { for } t>0,
$$

and it is nonincreasing in $t$, where $I=\operatorname{spt}^{+}(v)$.

Proof. First we assume that $v>0$ on $\mathbb{R}^{1}$. Let $L>0$ be such that $\operatorname{spt}\left(u_{0}\right) \subset(-L, L)$. Then we can choose $\delta>0$ sufficiently small so that

$$
0<u(t, \pm L)<v( \pm L) \quad \text { for } 0 \leq t \leq \delta .
$$

Since $u(0, x)<v(x)$ for $|x| \geq L$, the comparison principle implies

$$
u(t, x)-v(x)<0 \quad \text { for } 0 \leq t \leq \delta,|x| \geq L .
$$

Thus, by Lemma 2.3 , we have

$$
\mathcal{Z}(u(t, \cdot)-v)<\infty \quad \text { for } 0<t \leq \delta .
$$

Since $\mathcal{Z}(u(t, \cdot)-v)$ is nonincreasing in $t, 2.14$ holds for all $t>0$.

Next we consider the case where $v$ is a compactly supported nonnegative solution. Put $\left[L^{-}, L^{+}\right]:=\operatorname{spt}^{+}(v)$. Then we have

$$
u\left(t, L^{ \pm}\right)>v\left(L^{ \pm}\right)=0 \quad \text { for } t>0 .
$$

This and Lemma 2.3 yield (2.14). The lemma is proved.

\subsection{Property of symmetrically decreasing solutions}

Lemma 2.10. Let $v(x)$ be a positive solution of (1.3) such that

$$
v^{\prime}\left(x_{0}\right)=0, \quad v^{\prime}(x)<0 \quad \text { for } x>x_{0} .
$$

Then there exists a sequence $\left\{v_{n}\right\}_{n=1}^{\infty}$ of positive periodic solutions such that

$$
v_{n}^{\prime}\left(x_{0}\right)=0(n=1,2, \ldots), \quad v_{n}\left(x_{0}\right) \nearrow v\left(x_{0}\right) \text { as } n \rightarrow \infty .
$$

Proof. Clearly the solution $v(x)$ is symmetric with respect to $x=x_{0}$, and $f\left(v\left(x_{0}\right)\right)>0$. Put $a:=\lim _{|x| \rightarrow \infty} v(x)$ and $b=v\left(x_{0}\right)$. Then multiplying (1.3) by $v^{\prime}$ and integrating it over $x_{0}<x<\infty$, we obtain

$$
\int_{a}^{b} f(s) d s=0, \quad \int_{c}^{b} f(s) d s>0 \quad \text { for } c \in(a, b) .
$$

Choose $\varepsilon_{0}>0$ sufficiently small so that $f(s)>0$ for $s \in\left[b-\varepsilon_{0}, b\right]$. Then for any $\varepsilon \in\left(0, \varepsilon_{0}\right]$, we can find $a_{\varepsilon} \in(a, b-\varepsilon)$ such that

$$
\int_{a_{\varepsilon}}^{b-\varepsilon} f(s) d s=0, \quad \int_{c}^{b-\varepsilon} f(s) d s>0 \quad \text { for any } c \in\left(a_{\varepsilon}, b-\varepsilon\right) .
$$


As is easily seen, the solution $v_{\varepsilon}$ of 1.3 satisfying $v_{\varepsilon}\left(x_{0}\right)=b-\varepsilon, v_{\varepsilon}^{\prime}\left(x_{0}\right)=0$ is either a symmetrically decreasing solution converging to $a_{\varepsilon}$ as $|x| \rightarrow \infty$, or a periodic solution oscillating between $b-\varepsilon$ and $a_{\varepsilon}$, depending on whether $f\left(a_{\varepsilon}\right)=0$ or $f\left(a_{\varepsilon}\right) \neq 0$. Note that $a_{\varepsilon}$ can also be characterized as

$$
F\left(a_{\varepsilon}\right)=m_{\varepsilon}, \quad \text { where } \quad F(u):=\int_{u}^{b} f(s) d s, \quad m_{\varepsilon}:=F(b-\varepsilon) .
$$

By Sard's theorem, $F^{\prime}\left(a_{\varepsilon}\right)\left(=-f\left(a_{\varepsilon}\right)\right) \neq 0$ for almost every value of $m_{\varepsilon}$. It follows that this holds for a.e. $\varepsilon \in\left(0, \varepsilon_{0}\right]$, since $m_{\varepsilon}=F(b-\varepsilon)$ and $\frac{d}{d \varepsilon} F(b-\varepsilon)=-f(b-\varepsilon) \neq 0$. This means that $v_{\varepsilon}$ is a periodic solution for almost every $\varepsilon \in\left(0, \varepsilon_{0}\right]$. The lemma is proved.

\section{Proof of the convergence theorem}

\subsection{Basic properties of the $\omega$-limit set}

Let $u(t, x)$ be as given in Theorem 1.1. Denote by $\omega(u)$ the $\omega$-limit set of $u(t, \cdot)$ in the topology of $L_{\text {loc }}^{\infty}\left(\mathbb{R}^{1}\right)$. Thus a function $w(x)$ belongs to $\omega(u)$ if and only if there exists a sequence $0<t_{n} \nearrow \infty$ such that

$$
\lim _{n \rightarrow \infty} u\left(t_{n}, x\right)=w(x) \quad \text { locally uniformly in } \mathbb{R}^{1} .
$$

By local parabolic estimates, we see that the convergence (3.1) implies convergence in the $C^{2}\left(\mathbb{R}^{1}\right)$ topology. Thus the definition of $\omega(u)$ remains unchanged if the topology of $L_{\mathrm{loc}}^{\infty}\left(\mathbb{R}^{1}\right)$ is replaced by that of $C^{2}\left(\mathbb{R}^{1}\right)$.

It is well-known that $\omega(u)$ is compact and connected, and it is an invariant set. This means that for any $w \in \omega(u)$ there exists an entire orbit (namely a solution of 1.1 defined for all $t \in \mathbb{R}^{1}$ ) passing through $w$. Choosing a suitable sequence $0<t_{n} \nearrow \infty$, we can find such an entire solution $W(t, x)$ with $W(0, x)=w(x)$ as follows:

$$
u\left(t+t_{n}, x\right) \rightarrow W(t, x) \quad \text { as } n \rightarrow \infty .
$$

Here the convergence is understood in the $L_{\text {loc }}^{\infty}$ sense in $(t, x) \in \mathbb{R}^{1} \times \mathbb{R}^{1}$, but, by parabolic regularity, it takes place in the $C^{1,2}\left(\mathbb{R}^{1} \times \mathbb{R}^{1}\right)$ sense.

The following lemma is an immediate consequence of Lemma 2.1 .

Lemma 3.1. Let $w=w(x)$ be any element of $\omega(u)$. Then

$$
w^{\prime}(x) \geq 0 \quad \text { for } x<L_{1}, \quad w^{\prime}(x) \leq 0 \quad \text { for } x>L_{2} .
$$

Consequently, $\omega(u)$ contains no periodic solution of (1.3) and no symmetrically increasing solution.

The next lemma follows easily from Lemma 2.8 
Lemma 3.2. Let $x_{0}$ be the point defined in 2.11. Then any element $w$ of $\omega(u)$ satisfies $w^{\prime}\left(x_{0}\right)=0$.

Proof. By the definition of $x_{0}$ and Lemma 2.8, we have $\lim _{t \rightarrow \infty} \operatorname{sgn}\left(u_{x}(t, x)\right)=1$ for all $x<x_{0}$ except possibly $x=\left(L_{1}+L_{2}\right) / 2$, and there exists a sequence of points $x_{n} \searrow x_{0}$ such that $\lim _{t \rightarrow \infty} \operatorname{sgn}\left(u_{x}\left(t, x_{n}\right)\right)=-1$. Consequently,

$$
w^{\prime}(x) \geq 0 \quad \text { for } x<x_{0}, \quad w^{\prime}\left(x_{n}\right) \leq 0 \quad \text { for } n=1,2, \ldots
$$

This implies $w^{\prime}\left(x_{0}\right)=0$ and the lemma is proved.

Lemma 3.3. Let $v(x)$ be a bounded positive solution of (1.3), and let $w$ be any element of $\omega(u)$. Then

$$
\text { either } \quad w \equiv v \quad \text { or } \quad \mathcal{Z}(w-v)<\infty
$$

Proof. The lemma follows immediately from Lemma 2.9 and (2.6).

\subsection{Further properties of the $\omega$-limit set}

Lemma 3.4. Let $u$ be as in Theorem 1.1. Then $\omega(u)$ consists only of stationary solutions.

Proof. Let $w$ be any element of $\omega(u)$ and $W(t, x)$ be as given in 3.2p. Since $W$ is a nonnegative solution of the equation in 1.1 , and since $f(0)=0$, by the strong maximum principle we have either $W(t, x)>0$ for all $t \in \mathbb{R}^{1}, x \in \mathbb{R}^{1}$, or $W \equiv 0$. In the latter case we have $w \equiv 0$, so the conclusion of the lemma holds. In what follows we assume the former; thus $w>0$.

Let $x_{0}$ be as in Lemma 3.2 and let $v(x)$ be the solution of the following initial value problem:

$$
v^{\prime \prime}+f(v)=0, \quad v\left(x_{0}\right)=w\left(x_{0}\right), \quad v^{\prime}\left(x_{0}\right)=0 .
$$

Then $v$ is symmetric about $x=x_{0}$. Since $w\left(x_{0}\right)>0, v$ is either a positive solution of (1.3) in $\mathbb{R}^{1}$, or a solution of $(1.3)$ with compact positive support. Then by Lemma 2.9 . $\mathcal{Z}_{I}(u(t, \cdot)-v)<\infty$ for $t>0$, where $I:=\operatorname{spt}^{+}(v)$. Since this quantity is nonincreasing in $t$, it is constant for all large $t$. Consequently, by Lemma 2.3. the function $u(t, x)-v(x)$ has only simple zeros on $I$ for all large $t$. In view of this and the fact that

$$
\lim _{n \rightarrow \infty} u\left(t+t_{n}, x\right)-v(x)=W(t, x)-v(x) \quad \text { in } C^{1}\left(\mathbb{R}^{1} \times I\right),
$$

and applying Lemma 2.6, we see that for each $t \in \mathbb{R}^{1}$, either $W(t, x)-v(x) \equiv 0$ on $I$, or $W(t, x)-v(x)$ has only simple zeros on $I$. However, the latter is impossible for $t=0$, since $W(0, x)-v(x)=w(x)-v(x)$ has a degenerate zero at $x=x_{0}$. Consequently, $W(0, x) \equiv v(x)$, that is, $w$ is a stationary solution. The lemma is proved. 


\subsection{Completion of the proof of Theorem 1.1}

It remains to show that $\omega(u)$ consists of only one element. Suppose that it contains more than one element. Then, since $\omega(u)$ is connected and compact in the topology of $L_{\mathrm{loc}}^{\infty}\left(\mathbb{R}^{1}\right)$, and since every element $w$ of $\omega(u)$ satisfies $w^{\prime}\left(x_{0}\right)=0$ by Lemma 3.2. there exist $0 \leq \gamma^{-}<\gamma^{+}$such that $\omega(u)$ consists of solutions $v_{\alpha}\left(\alpha \in\left[\gamma^{-}, \gamma^{+}\right]\right)$of (1.3) defined by

$$
v_{\alpha}\left(x_{0}\right)=\alpha, \quad v_{\alpha}^{\prime}\left(x_{0}\right)=0 .
$$

By Lemma 3.1. for each $\alpha \in\left[\gamma^{-}, \gamma^{+}\right], v_{\alpha}$ is neither a periodic solution nor a symmetrically increasing solution. In view of this and Lemma 2.10, we see that, for each $\alpha \in\left(\gamma^{-}, \gamma^{+}\right], v_{\alpha}$ is not a symmetrically decreasing solution. Consequently, $v_{\alpha}$ is a constant solution for every $\alpha \in\left(\gamma^{-}, \gamma^{+}\right]$, that is, $v_{\alpha} \equiv \alpha$. By continuity, the same holds for $\alpha=\gamma^{-}$. Fix $\alpha$ with $\gamma^{-}<\alpha<\gamma^{+}$. Then, since $\gamma^{-}$belongs to $\omega(u)$, there is a sequence $t_{n} \nearrow \infty$ such that

$$
u\left(t_{n}, x\right) \rightarrow \gamma_{-} \quad \text { as } n \rightarrow \infty \text { locally uniformly in } \mathbb{R}^{1} .
$$

In view of this and [2.1], we see that

$$
u\left(t_{n}, x\right) \leq \alpha \quad \text { for } x \in \mathbb{R}^{1} \text { for sufficiently large } n .
$$

Then by the comparison principle, we have $u(t, x) \leq \alpha$ for all large $t>0$, which contradicts the assumption that $\gamma^{+}$belongs to $\omega(u)$. This contradiction proves that $\omega(u)$ is a singleton.

Finally, as we have already noted, the element of $\omega(u)$ is either a constant or a symmetrically decreasing solution by virtue of Lemma 3.1 The proof of Theorem 1.1 is complete.

\subsection{A further remark}

We remark that certain types of constant solutions of (1.3) can never belong to the $\omega$-limit set of $u$.

Lemma 3.5. Let $u, f$ be as in Theorem 1.1 and $\beta$ be a positive zero of $f$. Then $\beta \notin \omega(u)$ if one of the following conditions holds for some $\varepsilon>0$ :

(a) $f(s) \leq 0$ for $s \in[\beta-\varepsilon, \beta+\varepsilon], \quad$ (b) $(\beta-s) f(s)<0$ for $s \in[\beta-\varepsilon, \beta) \cup(\beta, \beta+\varepsilon]$.

Proof. Let us first assume (a). Suppose that there exists a sequence $t_{n} \nearrow \infty$ such that $u\left(t_{n}, x\right)$ converges to $\beta$ as $n \rightarrow \infty$ locally uniformly in $\mathbb{R}^{1}$. Then by $[2.2]$, there exists $t_{0} \geq 0$ such that

$$
u\left(t_{0}, x\right) \leq \beta+\varepsilon \quad \text { for } x \in \mathbb{R}^{1} .
$$

Let $v(t, x)$ be a solution of the following problem:

$$
v_{t}=v_{x x} \quad\left(t \geq t_{0}, x \in \mathbb{R}^{1}\right), \quad v\left(t_{0}, x\right)=\max \left\{u\left(t_{0}, x\right), \beta-\varepsilon\right\} \quad\left(x \in \mathbb{R}^{1}\right) .
$$


Then $\beta-\varepsilon \leq v(t, x) \leq \beta+\varepsilon$ for all $t \geq t_{0}, x \in \mathbb{R}^{1}$, and hence, by condition (a), $v$ is a supersolution of (1.1). Consequently $v \geq u$ for all $t \geq t_{0}$. On the other hand, by [2.3], $v\left(t_{0}, x\right)$ equals $\beta-\varepsilon$ for all large $|x|$. It follows that $v(t, x) \rightarrow \beta-\varepsilon$ as $t \rightarrow \infty$, and

$$
\beta=\lim _{t \rightarrow \infty} u(t, x) \leq \lim _{t \rightarrow \infty} v(t, x)=\beta-\varepsilon<\beta .
$$

This contradiction shows that $\lim _{t \rightarrow \infty} u \neq \beta$.

Next we assume (b). Then we easily see that there exists a positive periodic solution $v(x)$ of 1.3 satisfying $\min v<\beta<\max v$, which implies that $\mathcal{Z}(\beta-v)=\infty$. Hence we can apply Lemma 3.3 to conclude that $\beta \notin \omega(u)$. The lemma is proved.

\section{Sharp threshold results}

In this section we consider two specific classes of nonlinearities-those of the bistable type and those of the combustion type-and prove Theorems 1.3 and 1.4

\subsection{Bistable nonlinearity}

Throughout this subsection we assume that

$$
f \text { is locally Lipschitz and satisfies } 1.4 \text { and } 1.5 \text {. }
$$

Let $u$ be the solution of (1.1) with $u_{0}$ as in Theorem 1.1. Since $f(s)<0$ for $s>1$, a simple comparison argument shows that $u$ is bounded; hence, by Theorem 1.1, $u(t, x)$ converges to a solution of the stationary problem $(1.3)$ as $t \rightarrow \infty$. Recall that solutions of (1.3) which are constants or symmetrically decreasing can only be:

$$
0, \quad \theta_{0}, \quad 1, \quad U(x-a)\left(a \in \mathbb{R}^{1}\right),
$$

where $U$ is determined by $(1.8)$. Since $\theta_{0}$ intersects each periodic solution of (1.3) infinitely many times, we see from Lemma 3.3 that $\theta_{0} \notin \omega(u)$. Therefore

$$
\omega(u)=\{0\}, \quad\{1\} \quad \text { or } \quad\{U(\cdot-a)\} \text { for some } a \in \mathbb{R}^{1},
$$

where the $\omega$-limit set is defined with respect to the topology of $L_{\mathrm{loc}}^{\infty}\left(\mathbb{R}^{1}\right)$.

Lemma 4.1. Suppose that $\left(\Phi_{1}\right)$ through $\left(\Phi_{3}\right)$ hold. Then the set

$$
\Sigma_{0}:=\left\{\lambda>0: u_{\lambda}(t, x) \rightarrow 0 \text { as } t \rightarrow \infty \text { locally uniformly in } x \in \mathbb{R}^{1}\right\}
$$

is an open interval $\left(0, \lambda^{0}\right)$ for some $\lambda^{0} \in(0, \infty]$. 
Proof. We first observe that

$$
\lambda \in \Sigma_{0} \Leftrightarrow \max _{L_{1}^{\lambda} \leq x \leq L_{2}^{\lambda}} u_{\lambda}\left(t_{0}, x\right)<\theta_{0} \text { for some } t_{0}>0
$$

where $\left[L_{1}^{\lambda}, L_{2}^{\lambda}\right]=\left[\operatorname{spt}\left(\phi_{\lambda}\right)\right]$. Indeed, the assertion ' $\Rightarrow$ ' is obvious. To prove ' $\Leftarrow$ ', suppose that the right-hand side of (4.2) holds. Then, by (2.2), we have

$$
0 \leq u_{\lambda}\left(t_{0}, x\right) \leq \theta_{0}-\varepsilon \quad\left(x \in \mathbb{R}^{1}\right) \quad \text { for some } \varepsilon>0 .
$$

Since $f(s)<0$ for $s \in\left(0, \theta_{0}\right)$, we see from a comparison argument that $u_{\lambda}(t, x) \rightarrow 0$ as $t \rightarrow \infty$ uniformly in $x \in \mathbb{R}^{1}$, which proves 4.2).

We next show that $\lambda \in \Sigma_{0}$ if $\lambda>0$ is sufficiently small. Fix $t_{0}>0$ arbitrarily. By $\left(\Phi_{1}\right)$ and $\left(\Phi_{2}\right), \phi_{\lambda}$ remains uniformly bounded on $\mathbb{R}^{1}$ as $\lambda$ varies in a finite interval. This and $\left(\Phi_{3}\right)$ imply that $\phi_{\lambda} \rightarrow 0$ in $L^{p}\left(\mathbb{R}^{1}\right)$ for any $p>1$ as $\lambda \rightarrow 0$. By standard interior parabolic estimates, $u_{\lambda}\left(t_{0}, x\right) \rightarrow 0$ as $\lambda \rightarrow 0$ uniformly in $x \in\left[L_{1}^{\delta}, L_{2}^{\delta}\right]$ for each $\delta>0$; hence, by (4.2), we have $\lambda \in \Sigma_{0}$ for sufficiently small $\lambda>0$. In particular, $\Sigma_{0} \neq \emptyset$.

Put $\lambda^{0}:=\sup \Sigma_{0}$. Then $0<\lambda^{0} \leq \infty$, and the comparison theorem and ( $\left.\Phi_{2}\right)$ yield

$$
\left(0, \lambda^{0}\right) \subset \Sigma_{0} \text {. }
$$

It remains to show that $\lambda^{0} \notin \Sigma_{0}$ if $\lambda^{0}<\infty$. Suppose the contrary. Then the right-hand side of (4.2) holds for $\lambda=\lambda^{0}$ and some $t_{0}>0$. Then, since the mapping $\lambda \mapsto u_{\lambda}\left(t_{0}, \cdot\right)$ is continuous from $\left[0, \lambda^{0}+\delta\right]$ to $C\left(\left[L_{1}^{\lambda^{0}+\delta}, L_{2}^{\lambda^{0}+\delta}\right]\right)(\delta>0)$ by virtue of $\left(\Phi_{3}\right)$ and parabolic estimates, we see that the right-hand side of (4.2) holds for $\lambda=\lambda^{0}+\delta$ if $\delta>0$ is sufficiently small. This contradicts the definition of $\lambda^{0}$, and the lemma is proved.

The following lemma gives a sufficient condition for $\omega(u)=\{1\}$.

Lemma 4.2. For any given constant $\alpha \in\left(\theta_{0}, 1\right)$, there exists $L_{\alpha}>0$ depending on $\alpha, f$ such that the solution $u(t, x)$ of $\left[1.1\right.$ with initial data $\alpha \chi_{\left[-L_{\alpha}, L_{\alpha}\right]}$ converges to 1 as $t \rightarrow \infty$ locally uniformly in $x \in \mathbb{R}^{1}$.

Proof. If $f$ is $C^{1}$ and $f^{\prime}(0)<0, f^{\prime}(1)<0$ then the conclusion follows from Theorem 3.2 of $[\mathrm{FM}]$. Clearly we can choose a function $g \in C^{1}([0, \infty))$ such that

$$
\begin{aligned}
& f(s) \geq g(s) \quad \text { for } s \geq 0, \quad g(0)=g\left(\xi_{0}\right)=g\left(\xi_{1}\right)=0, \\
& g(s)<0 \quad \text { for } s \in\left(0, \xi_{0}\right) \cup\left(\xi_{1}, \infty\right), \quad g(s)>0 \quad \text { for } s \in\left(\xi_{0}, \xi_{1}\right), \\
& g^{\prime}(0)<0, \quad g^{\prime}\left(\xi_{1}\right)<0, \quad \int_{0}^{\xi_{1}} g(s) d s>0
\end{aligned}
$$

for some $\xi_{0} \in\left(0, \theta_{0}\right), \xi_{1} \in\left(\theta_{0}, 1\right)$. Let $v$ be the solution of the problem

$$
v_{t}=v_{x x}+g(v) \quad\left(t>0, x \in \mathbb{R}^{1}\right), \quad v(0, x)=\alpha \chi_{\left[-L_{0}, L_{0}\right]}(x) \quad\left(x \in \mathbb{R}^{1}\right) .
$$

Applying [FM, Theorem 3.2] to the above problem, we get $\lim _{t \rightarrow \infty} v(t, x)=\xi_{1}$ if $L_{0}$ is chosen sufficiently large. Since $f \geq g$, the comparison principle yields

$$
\lim _{t \rightarrow \infty} u(t, x) \geq \lim _{t \rightarrow \infty} v(t, x)=\xi_{1} .
$$

This and 4.1 imply $\omega(u)=\{1\}$. The lemma is proved. 
Lemma 4.3. Suppose that $\left(\Phi_{1}\right)$ through $\left(\Phi_{3}\right)$ hold. Then

$$
\Sigma_{1}:=\left\{\lambda>0: u_{\lambda}(t, x) \rightarrow 1 \text { as } t \rightarrow \infty \text { locally uniformly in } x \in \mathbb{R}^{1}\right\}
$$

is either empty or an open interval $\left(\lambda^{1}, \infty\right)$ for some $\lambda^{1}>0$.

Proof. Fix constants $\alpha \in\left(\theta_{0}, 1\right)$ and $L_{\alpha}>0$ as in Lemma 4.2 . Then

$$
\lambda \in \Sigma_{1} \Leftrightarrow \min _{-L_{\alpha} \leq x \leq L_{\alpha}} u_{\lambda}\left(t_{0}, x\right)>\alpha \text { for some } t_{0}>0 .
$$

Indeed, ' $\Rightarrow$ ' is obvious, and ' $\Leftarrow$ ' follows easily from Lemma 4.2 , the comparison principle and (4.1). Now assume $\Sigma_{1} \neq \emptyset$, and put $\lambda^{1}:=\inf \Sigma_{1}$. Then the comparison theorem and $\left(\Phi_{2}\right)$ imply

$$
\left(\lambda^{1}, \infty\right) \subset \Sigma_{1}
$$

It remains to show that $\lambda^{1} \notin \Sigma_{1}$; but this follows easily from (4.3), by an argument analogous to that in the last part of the proof of Lemma 4.1 The lemma is proved.

The following lemma shows the sharpness of transition between extinction and propagation.

Lemma 4.4. If $\lim _{t \rightarrow \infty} u_{\lambda^{*}}=U\left(x-a_{0}\right)$ for some $\lambda^{*}>0$ and $a_{0} \in \mathbb{R}^{1}$, then $\lim _{t \rightarrow \infty} u_{\lambda}$ $=1$ for every $\lambda>\lambda^{*}$.

We will prove the above assertion using the following lemma.

Lemma 4.5. Let $\phi_{1}, \phi_{2}$ be bounded nonnegative functions on $\mathbb{R}^{1}$ satisfying

$$
\phi_{1} \leq \phi_{2}, \quad \phi_{1} \not \equiv \phi_{2}, \quad\left[\operatorname{spt}\left(\phi_{2}\right)\right] \subset[-L, L]
$$

for some $L>0$, and let $u_{i}$ be the solution of 1.1) with $u_{0}=\phi_{i}, i=1,2$. Then there exist positive constants $t_{0}, \delta$ and $\varepsilon$ such that

$$
u_{2}(t+\delta, x)>u_{1}(t, x+a) \quad \text { for } t \geq t_{0}, x \in \mathbb{R}^{1} \text { and }|a| \leq \varepsilon .
$$

Proof. By Proposition 1.8, there exists $t_{0}>0$ such that $u_{i}(t, x)$ is increasing in $t$ for $t \in\left(0,2 t_{0}\right]$ and $|x| \geq 1 /\left(2 t_{0}\right)$. We may assume $L_{0}:=1 /\left(2 t_{0}\right)>L$.

By the strong maximum principle, we have $u_{2}(t, x)>u_{1}(t, x)$ for all $t>0$ and $x \in \mathbb{R}^{1}$. Thus, by uniform continuity, we can find constants $\delta \in\left(0, t_{0}\right]$ and $\varepsilon>0$ such that

$$
u_{2}\left(t_{0}+\delta, x\right)>u_{1}\left(t_{0}, x+a\right) \quad \text { for } x \in\left[-L_{0}, L_{0}\right],|a| \leq \varepsilon .
$$

Now the inequality $u_{2}(t, x)>u_{1}(t, x)$ and the monotonicity of $u_{2}\left(t, \pm L_{0}\right)$ in $t$ imply

$$
u_{2}(t+\delta, x)>u_{1}(t, x) \quad \text { for } t \in\left[0, t_{0}\right], x= \pm L_{0} .
$$

Since $L_{0}>L, u_{1}(t, x)$ is continuous up to $t=0$ in a neighborhood of $x= \pm L_{0}$. Therefore, if we choose a sufficiently small $\varepsilon>0$, then from (4.5) we see that

$$
u_{2}(t+\delta, x)>u_{1}(t, x+a) \quad \text { for } t \in\left[0, t_{0}\right], x= \pm L_{0},|a| \leq \varepsilon .
$$


Also, since $u_{1}(0, x)=0$ for $|x|>L$, we have

$$
u_{2}(\delta, x)>u_{1}(0, x+a) \quad \text { for }|x| \geq L_{0},|a| \leq \varepsilon,
$$

provided that $\varepsilon$ is chosen small enough so that $\varepsilon<L_{0}-L$. Combining this and (4.6), and applying the comparison principle, we obtain

$$
u_{2}\left(t_{0}+\delta, x\right)>u_{1}\left(t_{0}, x+a\right) \text { for }|x| \geq L_{0} .
$$

This and 4.4 yield

$$
u_{2}\left(t_{0}+\delta, x\right)>u_{1}\left(t_{0}, x+a\right) \quad \text { for } x \in \mathbb{R}^{1},|a| \leq \varepsilon .
$$

The conclusion of the lemma now follows from this and the comparison principle.

Proof of Lemma 4.4 Let $\lambda^{*}<\lambda$ be as in the lemma. Then, by Lemma 4.5, we have

$$
u_{\lambda}(t+\delta, x)>u_{\lambda^{*}}(t, x+a) \quad \text { for } t \geq t_{0}, x \in \mathbb{R}^{1} \text { and }|a| \leq \varepsilon .
$$

Put $v(x):=\lim _{t \rightarrow \infty} u_{\lambda}(t, x)$. Then $v$ satisfies

$$
v(x) \geq U\left(x-a_{0}+a\right) \quad \text { for } x \in \mathbb{R}^{1},|a| \leq \varepsilon .
$$

The only solution of (1.3) satisfying this is $v=1$. The lemma is proved.

Now we are ready to prove the main theorem of this subsection.

Proof of Theorem 1.3 By Lemma 4.1, we have $\Sigma_{0}=\left(0, \lambda^{0}\right)$ for some $\lambda^{0} \in(0, \infty)$. If $\lambda^{0}=\infty$, then the alternative (a) of the theorem holds. On the other hand, if $\lambda^{0}<\infty$, then by Lemma $4.3 . \lambda^{0} \notin \Sigma_{0} \cup \Sigma_{1}$, hence

$$
u_{\lambda^{0}}(t, x) \rightarrow U\left(x-a_{0}\right) \quad \text { as } t \rightarrow \infty \quad \text { for some } a_{0} \in\left[L_{1}, L_{2}\right] .
$$

By Lemma 4.4 , we have $\lambda \in \Sigma_{1}$ for all $\lambda>\lambda^{0}$. The proof of the theorem is complete.

Remark 4.6. In the bistable case $\left(\mathrm{f}_{B}\right)$, the convergence results of Theorem 1.1 can be proved more easily by using the energy functional

$$
J(u(t, \cdot)):=\int_{\mathbb{R}^{1}}\left[\frac{1}{2} u_{x}^{2}(t, x)-\int_{0}^{u(t, x)} f(s) d s\right] d x .
$$

Indeed, if $\lim _{t \rightarrow \infty} u(t, x) \neq 1$, then by Lemmas 2.1 and 4.2 there exists $L>0$ with $\operatorname{spt}\left(u_{0}\right) \subset[-L, L]$ such that $u(t, x) \leq \theta^{*}$ for all $t \geq 0,|x| \geq L$. This and the comparison principle imply

$$
u(t, x) \leq U(x+L) \quad \text { for } x \leq-L, \quad u(t, x) \leq U(x-L) \quad \text { for } x \geq L .
$$

From the above estimates one sees that $J(u(t, \cdot))$ is bounded from below as $t \rightarrow \infty$; hence $\omega(u)$ is contained in the set of nonnegative solutions of $(1.3)$ decaying to 0 as $|x| \rightarrow \infty$. One can then use Lemma 2.8 to conclude that either $\omega(u)=\{0\}$ or $\omega(u)=$ $\{U(\cdot-a)\}$ for some $a \in \mathbb{R}^{1}$. 


\subsection{Combustion nonlinearity}

In this subsection we assume that

$f$ is locally Lipschitz and satisfies 1.6 and (1.7).

Let $u$ be as in Theorem 1.4 Since $f(s) \leq 0$ for $s \in[1, \infty)$, we see by a comparison argument that $u$ remains bounded as $t \rightarrow \infty$. Thus, by Theorem 1.1, $u$ converges to a stationary solution as $t \rightarrow \infty$. As we have mentioned in the introduction, any nonnegative solution of (1.3) is constant. This and Lemma 3.5 imply

$$
\omega(u)=\{0\}, \quad\left\{\theta_{0}\right\} \quad \text { or }\{1\} .
$$

Our proof of Theorem 1.4 is for the most part analogous to that of Theorem 1.3 in the previous subsection, though differences arise in some details, particularly in the proof of Lemma4.10 (sharpness of the transition).

Lemma 4.7. Suppose that $\left(\Phi_{1}\right)$ through $\left(\Phi_{3}\right)$ hold. Then the set

$$
\Sigma_{0}:=\left\{\lambda>0: u_{\lambda}(t, x) \rightarrow 0 \text { as } t \rightarrow \infty \text { locally uniformly in } x \in \mathbb{R}^{1}\right\}
$$

is an open interval $\left(0, \lambda^{0}\right)$ for some $\lambda^{0} \in(0, \infty]$.

Proof. We first prove (4.2). The assertion ' $\Rightarrow$ ' is obvious. To prove ' $\Leftarrow$ ', suppose that the right-hand side of 4.2$]$ holds. Then, by [2.2], we have

$$
0 \leq u_{\lambda}\left(t_{0}, x\right) \leq \theta_{0}-\varepsilon \quad\left(x \in \mathbb{R}^{1}\right) \quad \text { for some } \varepsilon>0 .
$$

Since $\theta_{0}-\varepsilon$ is a stationary solution, the comparison principle yields

$$
\lim _{t \rightarrow \infty} u(t, x) \leq \theta_{0}-\varepsilon .
$$

This and (4.8) imply $u(t, x) \rightarrow 0$ as $t \rightarrow \infty$, establishing (4.2). The rest of the proof is identical to that of Lemma 4.1 .

Lemma 4.8. For any given constant $\alpha \in\left(\theta_{0}, 1\right)$, there exists $L_{\alpha}>0$ depending on $\alpha, f$ such that the solution $u(t, x)$ of 11.1 with initial data $\alpha \chi_{\left[-L_{\alpha}, L_{\alpha}\right]}$ converges to 1 as $t \rightarrow \infty$ locally uniformly in $x \in \mathbb{R}^{\text {? }}$.

Proof. By modifying the value of $f$ in the range $\left(0, \theta_{0}\right) \cup(1, \infty)$, we can construct a function $\tilde{f}$ satisfying $\left(\mathrm{f}_{B}\right)$ and

$$
\tilde{f}(s) \leq f(s) \quad \text { for } 0 \leq s \leq 1 .
$$

Let $\tilde{u}(t, x)$ be the solution of the problem

$$
\tilde{u}_{t}=\tilde{u}_{x x}+\tilde{f}(\tilde{u}) \quad\left(t>0, x \in \mathbb{R}^{1}\right), \quad \tilde{u}(0, x)=\alpha \chi_{\left[-L_{\alpha}, L_{\alpha}\right]}(x) \quad\left(x \in \mathbb{R}^{1}\right) .
$$

By Lemma 4.2 $\tilde{u}(t, x) \rightarrow 1$ as $t \rightarrow \infty$, if $L_{\alpha}$ is chosen large enough. Since $0 \leq \tilde{u} \leq 1$ and $\tilde{f}(s) \leq f(s)$ for $s \in[0,1], \tilde{u}$ is a subsolution for (1.1). Therefore, $\lim _{t \rightarrow \infty} u(t, x) \geq$ $\lim _{t \rightarrow \infty} \tilde{u}(t, x)=1$. By [4.8), $\lim _{t \rightarrow \infty} u(t, x)=1$. The lemma is proved. 
Lemma 4.9. Suppose that $\left(\Phi_{1}\right)$ through $\left(\Phi_{3}\right)$ hold. Then

$$
\Sigma_{1}:=\left\{\lambda>0: u_{\lambda}(t, x) \rightarrow 1 \text { as } t \rightarrow \infty \text { locally uniformly in } x \in \mathbb{R}^{1}\right\}
$$

is either empty or an open interval $\left(\lambda^{1}, \infty\right)$ for some $\lambda^{1}>0$.

Proof. Fix constants $\alpha \in\left(\theta_{0}, 1\right)$ and $L_{\alpha}>0$ as in Lemma 4.8. Then (4.3) holds. Indeed, ' $\Rightarrow$ ' is obvious, and ' $\Leftarrow$ ' is also clear from Lemma 4.8 , the comparison principle and (4.8). Once we have 4.3, the rest of the proof is identical to that of Lemma 4.3

The following lemma shows the sharpness of transition between extinction and propagation.

Lemma 4.10. If $\lim _{t \rightarrow \infty} u_{\lambda^{*}}=\theta_{0}$ for some $\lambda^{*}>0$, then $\lim _{t \rightarrow \infty} u_{\lambda}=1$ for every $\lambda>\lambda^{*}$.

The above lemma will follow easily from the next lemma.

Lemma 4.11. Let $\phi_{1}, \phi_{2}$ be bounded nonnegative functions on $\mathbb{R}^{1}$ satisfying

$$
\phi_{1} \leq \phi_{2}, \quad \phi_{1} \not \equiv \phi_{2}, \quad\left[\operatorname{spt}\left(\phi_{2}\right)\right] \subset[-L, L]
$$

for some $L>0$, and let $u_{i}$ be the solution of (1.1) with $u_{0}=\phi_{i}, i=1,2$. Assume that

$$
\left\|u_{1}(t, \cdot)\right\|_{L^{\infty}} \leq \theta_{0}+\delta_{0} / 2 \quad \text { for all large } t \geq 0
$$

where $\theta_{0}$ is the constant in (1.7). Then there exist constants $T>0, \delta>0$ and $\sigma \in(0,1)$ such that

$$
u_{2}(t+\delta, x)>\sigma^{-1} u_{1}(\sigma t, \sqrt{\sigma} x) \quad \text { for } x \in \mathbb{R}^{1}, t \geq T .
$$

Proof. Choose $T \geq 1$ such that

$$
\left\|u_{1}(t, \cdot)\right\|_{L^{\infty}} \leq \theta_{0}+\delta_{0} / 2 \quad \text { for } t \geq T-1 .
$$

Arguing as in Lemma 4.5, we find that there exists $\delta>0$ such that

$$
u_{2}(T+\delta, x)>u_{1}(T, x) \quad \text { for } x \in \mathbb{R}^{1} .
$$

Now, given a constant $\sigma \in(0,1)$, we define

$$
v^{\sigma}(t, x):=\sigma^{-1} u_{1}(\sigma t, \sqrt{\sigma} x) .
$$

Then, by [4.9), we can choose $\sigma_{0} \in(0,1)$ close enough to 1 so that

$$
\left\|v^{\sigma}(t, \cdot)\right\|_{L^{\infty}} \leq \theta_{0}+\delta_{0} \quad \text { for every } t \geq T, \sigma \in\left[\sigma_{0}, 1\right] .
$$

Observe that $v^{\sigma}$ satisfies the equation

$$
v_{t}^{\sigma}=v_{x x}^{\sigma}+f\left(\sigma v^{\sigma}\right) .
$$

By (4.11), (1.6) and (1.7), we have $f\left(\sigma v^{\sigma}\right) \leq f\left(v^{\sigma}\right)$, therefore $v^{\sigma}$ is a subsolution of 1.1 for $t \geq T$. 
To complete the proof of the lemma, in view of the comparison principle, we only have to show that

$$
u_{2}(T+\delta, x) \geq v^{\sigma}(T, x) \quad \text { for } x \in \mathbb{R}^{1} .
$$

To do so, we first derive the estimate

$$
\lim _{|x| \rightarrow \infty} \frac{u_{2}(T+\delta, x)}{v^{\sigma}(T, x)}=\infty \quad \text { uniformly in } \sigma \in\left[\sigma_{0}, 1\right]
$$

Since $f$ is locally Lipschitz continuous with $f(0)=0$ and since $u_{1}, u_{2}$ are bounded, there exists a constant $M \geq 0$ such that $\left|f\left(u_{i}\right)\right| \leq M u_{i}(i=1,2)$. Therefore

$$
e^{-M t} \bar{u}_{i}(t, x) \leq u_{i}(t, x) \leq e^{M t} \bar{u}_{i}(t, x) \quad(i=1,2)
$$

where $\bar{u}_{i}$ is the solution of $u_{t}=u_{x x}$ with initial data $\phi_{i}, i=1,2$. Consequently,

$$
\frac{u_{2}(T+\delta, x)}{v^{\sigma}(T, x)}=\frac{u_{2}(T+\delta, x)}{\sigma^{-1} u_{1}(\sigma T, \sqrt{\sigma} x)} \geq C_{\sigma} \frac{\bar{u}_{2}(T+\delta, x)}{\bar{u}_{1}(\sigma T, \sqrt{\sigma} x)} \geq C_{\sigma} \frac{\bar{u}_{2}(T+\delta, x)}{\bar{u}_{2}(\sigma T, \sqrt{\sigma} x)},
$$

where $C_{\sigma}:=\sigma e^{-M(\sigma T+T+\delta)}$. The last term is calculated as

$$
\tilde{C}_{\sigma} \int_{-L}^{L} \exp \left(-\frac{(x-y)^{2}}{4(T+\delta)}\right) \phi_{2}(y) d y / \int_{-L}^{L} \exp \left(-\frac{\left(x-\sqrt{\sigma^{-1}} y\right)^{2}}{4 T}\right) \phi_{2}(y) d y,
$$

with $\tilde{C}_{\sigma}=C_{\sigma} \sqrt{\sigma T /(T+\delta)}$. Since $\delta>0$, the above quantity tends to $\infty$ as $|x| \rightarrow \infty$, thus proving (4.13). Consequently, there exists $R>0$ such that

$$
u_{2}(T+\delta, x) \geq v^{\sigma}(T, x) \quad \text { for }|x| \geq R, \sigma \in\left[\sigma_{0}, 1\right] .
$$

Choosing $\sigma$ sufficiently close to 1 , we also have, by virtue of 4.10,

$$
u_{2}(T+\delta, x) \geq v^{\sigma}(T, x) \text { for }|x| \leq R .
$$

This establishes 4.12, completing the proof of the lemma.

Proof of Lemma 4.10. Let $\lambda^{*}<\lambda$ be as in the lemma. The convergence $u_{\lambda^{*}}(t, x) \rightarrow \theta_{0}$ as $t \rightarrow \infty$ and 2.2 imply that there exists $T>1$ such that

$$
\left\|u_{\lambda^{*}}(t, \cdot)\right\|_{L^{\infty}} \leq \theta_{0}+\delta_{0} / 2 \text { for } t \geq T-1 .
$$

Therefore, by Lemma 4.11, we have, for some $\sigma \in(0,1)$,

$$
u_{\lambda}(t+\delta, x)>\sigma^{-1} u_{\lambda^{*}}(\sigma t, \sqrt{\sigma} x) \quad \text { for } x \in \mathbb{R}^{1}, t \geq T .
$$

Letting $t \rightarrow \infty$, we obtain

$$
\lim _{t \rightarrow \infty} u_{\lambda}(t, x) \geq \sigma^{-1} \theta_{0} .
$$

In view of 4.8 and $\sigma^{-1} \theta_{0}>\theta_{0}$, we see that $\lim _{t \rightarrow \infty} u_{\lambda}=1$. The lemma is proved.

Proof of Theorem 1.4 The proof is identical to that of Theorem 1.3 , except that Lemmas 4.1, 4.3 and 4.4 are now replaced by Lemmas 4.7, 4.9 and 4.10. 
Remark 4.12. If $\theta_{0}=0$, the situation is slightly different. Lemma 4.9 and its proof remain valid, hence the alternatives (a), (b) of Theorem 1.4 still hold, except that we may have $\lambda^{*}=0$ in the alternative (b):

$$
\lim _{t \rightarrow \infty} u_{\lambda}(t, x)=\left\{\begin{array}{lll}
0 & \text { uniformly in } \mathbb{R}^{1} & \left(0 \leq \lambda \leq \lambda^{*}\right), \\
1 & \text { locally uniformly in } \mathbb{R}^{1} & \left(\lambda>\lambda^{*}\right),
\end{array}\right.
$$

for some $\lambda^{*} \geq 0$. Whether $\lambda^{*}=0$ (known as the hair-trigger effect) or $\lambda^{*}>0$ depends on the behavior of $f(s)$ near $s=0$. More precisely, $\lambda^{*}=0$ if $f(s) \geq C s^{p}$ for some $p<3$ and all small $s>0$, while $\lambda^{*}>0$ if $f(s) \leq C s^{p}$ for some $p>3$ and all small $s>0$; see Section 3 of [AW2] and the Remark after Theorem 1 of [Z] for further details. Thus we may call $p=3$ a threshold value. For problems in $\mathbb{R}^{N}$, the threshold value of $p$ is $1+2 / N$ (see [AW2]).

\section{Proof of Proposition 1.8}

Since $g$ is locally Lipschitz continuous and $u_{0} \in L^{\infty}\left(\mathbb{R}^{N}\right)$, by the standard parabolic theory, $u(t, x)$ is defined for $x \in \mathbb{R}^{N}$ on some maximal time interval of existence $\left(0, T\left(u_{0}\right)\right)$, with $0<T\left(u_{0}\right) \leq \infty$. Moreover, for each $T \in\left(0, T\left(u_{0}\right)\right)$ there exists $C_{T}>0$ such that $0<u(t, x) \leq C_{T}$ for all $t \in(0, T]$ and $x \in \mathbb{R}^{N}$.

Fix $T \in\left(0, T\left(u_{0}\right)\right)$. Then there exists $M>0$ such that

$$
\left|g\left(s_{1}\right)-g\left(s_{2}\right)\right| \leq M\left|s_{1}-s_{2}\right| \quad \text { for all } s_{1}, s_{2} \in\left[0, C_{T}\right] .
$$

Therefore, due to $g(0)=0$,

$$
-M s \leq g(s) \leq M s \quad \text { for all } s \in\left[0, C_{T}\right] .
$$

Let $\bar{u}(t, x)$ be the solution of the following problem:

$$
\bar{u}_{t}=\Delta \bar{u} \quad \text { for }(t, x) \in \mathbb{R}_{+} \times \mathbb{R}^{N}, \quad \bar{u}(0, x)=u_{0}(x) \quad \text { for } x \in \mathbb{R}^{N} .
$$

Then

$$
\bar{u}(t, x)=\int_{\Omega}(4 \pi t)^{-N / 2} \exp \left(-\frac{|x-y|^{2}}{4 t}\right) u_{0}(y) d y,
$$

where $\Omega=\operatorname{spt}\left(u_{0}\right)$. One easily checks that $e^{M t} u$ and $e^{-M t} u$ are, respectively, super- and subsolutions of (5.1) for $(t, x) \in(0, T] \times \mathbb{R}^{N}$; hence

$$
e^{-M t} \bar{u}(t, x) \leq u(t, x) \leq e^{M t} \bar{u}(t, x) \quad \text { for all }(t, x) \in(0, T] \times \mathbb{R}^{N} .
$$

Now we choose an arbitrary sequence $\left(t_{n}, x_{n}\right) \in(0, \infty) \times \mathbb{R}^{N}$ satisfying

$$
t_{n} \rightarrow 0, \quad\left|x_{n}\right| \rightarrow \infty \quad \text { as } n \rightarrow \infty
$$

and define

$$
w_{n}(s, z)=\frac{u\left(t_{n}+\varepsilon_{n}^{2} s, x_{n}+\varepsilon_{n} z\right)}{\bar{u}\left(t_{n}, x_{n}\right)}, \quad \bar{w}_{n}(s, z)=\frac{\bar{u}\left(t_{n}+\varepsilon_{n}^{2} s, x_{n}+\varepsilon_{n} z\right)}{\bar{u}\left(t_{n}, x_{n}\right)},
$$


where $\varepsilon_{n}=t_{n} /\left|x_{n}\right|$. These functions are well-defined if $0<t_{n}+\varepsilon_{n}^{2} s<T$, or, equivalently, if $-\left|x_{n}\right|^{2} / t_{n}<s<\left(T-t_{n}\right)\left|x_{n}\right|^{2} / t_{n}^{2}$. By [5.3), we have

$$
e^{-M\left(t_{n}+\varepsilon_{n}^{2} s\right)} \bar{w}_{n}(s, z) \leq w_{n}(s, z) \leq e^{M\left(t_{n}+\varepsilon_{n}^{2} s\right)} \bar{w}_{n}(s, z)
$$

as long as $0<t_{n}+\varepsilon_{n}^{2} s \leq T$. Using the expression 5.2 , we obtain

$$
\bar{w}_{n}(s, z)=\left(1+\frac{\varepsilon_{n}}{\left|x_{n}\right|} s\right)^{-N / 2} \frac{\int_{\Omega} \exp \left(-\frac{\left|x_{n}+\varepsilon_{n} z-y\right|^{2}}{4\left(t_{n}+\varepsilon_{n}^{2} s\right)}\right) u_{0}(y) d y}{\int_{\Omega} \exp \left(-\frac{\left|x_{n}-y\right|^{2}}{4 t_{n}}\right) u_{0}(y) d y} .
$$

Considering that $\Omega$ is bounded, a careful calculation shows that

$$
\frac{\left|x_{n}-y\right|^{2}}{4 t_{n}}-\frac{\left|x_{n}+\varepsilon_{n} z-y\right|^{2}}{4\left(t_{n}+\varepsilon_{n}^{2} s\right)}=\frac{s}{4}-\frac{1}{2} e_{n} \cdot z+O\left(\left|x_{n}\right|^{-1}\right),
$$

as long as $s, z, y$ vary in a bounded region, where $e_{n}=x_{n} /\left|x_{n}\right|$; we thus obtain

$$
\bar{w}_{n}(s, z)=\exp \left(\frac{s}{4}-\frac{1}{2} e_{n} \cdot z\right)+O\left(\left|x_{n}\right|^{-1}\right) .
$$

By passing to a subsequence, we may assume that $e_{n} \rightarrow \theta \in S^{N-1}$. This and (5.5) yield

$$
w_{n}(s, z)=\exp \left(\frac{s}{4}-\frac{1}{2} \theta \cdot z\right)+O\left(\left|x_{n}\right|^{-1}\right)+O\left(t_{n}\right),
$$

uniformly as $n \rightarrow \infty$ and as $(s, z)$ varies in a bounded subset of $\mathbb{R}^{1} \times \mathbb{R}^{N}$.

Observe that $w_{n}$ satisfies the equation

$$
\left(w_{n}\right)_{s}=\Delta_{z}\left(w_{n}\right)+\frac{\varepsilon_{n}^{2}}{\alpha_{n}} g\left(\alpha_{n} w_{n}\right) \quad\left(\text { where } \alpha_{n}:=\bar{u}\left(t_{n}, x_{n}\right)\right)
$$

in the region where $0<t_{n}+\varepsilon_{n}^{2} s \leq T, z \in \mathbb{R}^{N}$ and that the functions

$$
w \mapsto \frac{\varepsilon_{n}^{2}}{\alpha_{n}} g\left(\alpha_{n} w\right) \quad(n=1,2, \ldots)
$$

are Lipschitz continuous, uniformly in $n$, as long as $0 \leq w \leq C_{T} / \alpha_{n}$. They also converge to 0 uniformly for $w$ in any bounded interval $[0, C]$. Consequently, by interior parabolic estimates (see [LSU] or $[\overline{\mathrm{L}}]$ ), we see that $\left\{w_{n}\right\}$ is bounded in $C^{1+\mu / 2,2+\mu}(\bar{\Omega})$ for any bounded domain $\Omega \subset \mathbb{R}^{1} \times \mathbb{R}^{N}$. Therefore we can use a standard diagonal process to extract a further subsequence, still denoted by $\left\{w_{n}\right\}$, such that $w_{n}$ converges to some $w_{0}$ in the sense of $C^{1,2}\left(\mathbb{R}^{1} \times \mathbb{R}^{N}\right)$. By [5.6, necessarily

$$
w_{0}(s, z)=\exp \left(\frac{s}{4}-\frac{1}{2} \theta \cdot z\right)
$$


Hence

$$
\lim _{n \rightarrow \infty} \frac{\partial}{\partial s} w_{n}(s, z)=\frac{\partial}{\partial s} w_{0}(s, z)=\frac{1}{4} w_{0}(s, z)
$$

locally uniformly in $(s, z)$. It follows that

$$
\lim _{n \rightarrow \infty} \frac{t_{n}^{2}}{\left|x_{n}\right|^{2}} \frac{u_{t}\left(t_{n}, x_{n}\right)}{\bar{u}\left(t_{n}, x_{n}\right)}=\lim _{n \rightarrow \infty} \frac{\partial}{\partial s} w_{n}(0,0)=\frac{1}{4} .
$$

The assertion (1.12) now follows from this and (5.3), since $\left(t_{n}, x_{n}\right)$ is an arbitrary sequence in $(0, \infty) \times \mathbb{R}^{N}$ satisfying (5.4). This completes the proof of Proposition 1.8 .

\section{Nonlinearity with a jumping discontinuity}

In this section we consider the combustion and the bistable type nonlinearities with a jumping discontinuity at $u=1$ and show that all the previous main results continue to hold.

\subsection{The combustion case}

We begin with the case where $f$ satisfies ( $f_{C}$ ) except that it has the following jumping discontinuity at $u=1$ :

$$
f(1-0)>0 \geq f(1+0) .
$$

Such a case often occurs in applications (see $[\mathrm{BKS}]$ ). We will show that for such $f$, the conclusions of Theorems 1.1 and 1.4 remain valid. The same is true for the following type of discontinuity:

$$
f(1-0)=0>f(1+0) .
$$

Since this latter case can be treated precisely the same way (and in some cases more easily), we will focus on 6.1. Thus we assume that

$$
\left(\hat{\mathrm{f}}_{C}\right)\left\{\begin{array}{l}
f \text { is Lipschitz in }[0,1) \text { and locally Lipschitz in }[1, \infty) \\
f \text { satisfies } 1.6,1.7 \text { and } 6.1 .
\end{array}\right.
$$

Note that the jumping discontinuity in $f$ can create a situation that is markedly different from the regular case. For example, some solutions can take the value 1 on an open subset of $\mathbb{R}_{+} \times \mathbb{R}^{1}$ without being identically equal to 1 . This means that the zeros of $u-1$ are not necessarily isolated, and moreover degenerate zeros of $u-1$ do not necessarily disappear instantly. Therefore part of our previous argument that relied on Lemma 2.3 has to be modified.

Let us first look at what essential properties of $f$ are retained. Since the discontinuity is away from $u=0$, and $f(u)$ jumps down when $u$ increases across $u=1$, it is easy to see that for any given $C>0$ there exists $M=M_{C}>0$ such that, for $0 \leq u \leq v \leq C$,

$$
-M u \leq f(u) \leq M u, \quad f(v)-f(u) \leq M(v-u) .
$$

As we will see below, thanks to 6.2, most of our previous arguments for locally Lipschitz continuous $f$ carry over to the current discontinuous case. 
Lemma 6.1. Let the above assumptions on $f$ be satisfied and $u_{0} \in L^{\infty}\left(\mathbb{R}^{1}\right)$ be nonnegative. Then (1.1) has a unique (weak) solution $u$, which is defined for all $t>0$. Furthermore, if $u_{0}$ has compact support, then

$$
\begin{cases}\limsup _{t \rightarrow \infty} u(t, x) \leq 1 & \text { for fixed } x \in \mathbb{R}^{1}, \\ \lim _{|x| \rightarrow \infty} u(t, x)=0 & \text { uniformly in } t \in[0, T],\end{cases}
$$

where $T>0$ is an arbitrary positive constant.

Proof. For $\varepsilon>0$ small, we define a Lipschitz approximation of $f(u)$ as follows:

$$
f_{\varepsilon}(u)= \begin{cases}f(u), & u \in[0,1), \\ f(1-0)+\varepsilon^{-1}[f(1+\varepsilon)-f(1-0)](u-1), & u \in[1,1+\varepsilon], \\ f(u), & u>1+\varepsilon .\end{cases}
$$

Clearly $f_{\varepsilon}(u) \geq f(u)$ for all $u \geq 0$ and all sufficiently small $\varepsilon>0$. Furthermore, $f_{\varepsilon} \rightarrow f$ in $L_{\text {loc }}^{p}\left(\mathbb{R}_{+}\right)$for any $p>1$ as $\varepsilon \rightarrow 0$.

Let $u_{\varepsilon}(t, x)$ denote the solution of the equation

$$
u_{t}=u_{x x}+f_{\varepsilon}(u)
$$

on $\mathbb{R}_{+} \times \mathbb{R}^{1}$ with initial data $u_{0}(x)$. Then $u_{\varepsilon}(t, x)$ is defined for all $t>0$. Since $f_{\varepsilon}$ is nondecreasing in $\varepsilon$, the comparison argument shows that $u_{\varepsilon}$ is nondecreasing in $\varepsilon$. Therefore the pointwise limit

$$
u(t, x):=\lim _{\varepsilon \rightarrow 0} u_{\varepsilon}(t, x)
$$

exists for every $(t, x) \in[0, \infty) \times \mathbb{R}^{1}$. By standard parabolic estimates, this convergence takes place in $C_{\text {loc }}^{(1+\mu) / 2,1+\mu}\left(\mathbb{R}_{+} \times \mathbb{R}^{1}\right)$ for any $\mu \in(0,1)$. Hence $u$ is a weak solution of (1.1).

Next we prove the uniqueness assertion. Suppose that $u$ and $v$ are both weak solutions of 1.1. Then, since $f(0)=0$ and $f(s) \leq 0$ for $s>1$, we see by the maximum principle that both $u$ and $v$ are bounded. We claim that $u \leq v$ for all $t>0$ and $x \in \mathbb{R}^{1}$. Suppose the contrary. Then $\Omega:=\left\{(t, x) \in(0, \infty) \times \mathbb{R}^{1}: u(t, x)>v(t, x)\right\}$ is a nonempty open set. By 6.2], there exists a constant $M_{0}>0$ such that

$$
f(u(t, x))-f(v(t, x)) \leq M_{0}(u(t, x)-v(t, x))
$$

whenever $u(t, x)>v(t, x)$. This and the fact that $u=v$ at $t=0$ imply that $w:=u-v$ satisfies (in the weak sense)

$$
\begin{cases}w_{t}-w_{x x} \leq M_{0} w, & (t, x) \in \Omega \\ w=0, & (t, x) \in \partial \Omega\end{cases}
$$

From the maximum principle (of the Phragmén-Lindelöf type) we deduce $w \leq 0$ in $\Omega$, a contradiction to the definition of $\Omega$. Hence $u \leq v$. Similarly $v \leq u$. This implies $u \equiv v$, and the uniqueness assertion is proved. 
Finally, suppose $u_{0}$ has compact support. Then by Theorems 1.1, 1.4 and the definition of $f_{\varepsilon}$, we have $\lim _{t \rightarrow \infty} u_{\varepsilon}(t, x) \leq 1_{\varepsilon}$, where $1_{\varepsilon} \in(1,1+\varepsilon]$ is the minimal zero of $f_{\varepsilon}$ in $[1,1+\varepsilon]$. This and the inequality $0 \leq u \leq u_{\varepsilon}$ imply

$$
\limsup _{t \rightarrow \infty} u(t, x) \leq \lim _{t \rightarrow \infty} u_{\varepsilon}(t, x) \leq 1_{\varepsilon} .
$$

Letting $\varepsilon \rightarrow 0$, we obtain the first assertion in 6.3.

By (6.2), $-M s \leq f(s) \leq M s$ for all $s \in\left[0, \sup u_{\varepsilon}\right]$ and some $M>0$. Hence we can deduce $(2.3)$ as before. This proves the second assertion in 6.3 . The proof of the lemma is now complete.

As we have mentioned above, the unique weak solution of (1.1) belongs to the space $C_{\text {loc }}^{(1+\mu) / 2,1+\mu}\left(\mathbb{R}_{+} \times \mathbb{R}^{1}\right)$ for any $0<\mu<1$.

We have the following weaker version of Lemma 2.1 .

Lemma 6.2. Let $u(t, x)$ be the weak solution of (1.1) as in Lemma 6.1 and put [ $\left.\operatorname{spt}\left(u_{0}\right)\right]$ $=\left[L_{1}, L_{2}\right]$. Then

$$
u_{x} \geq 0 \text { for } x<L_{1}, t>0 ; \quad u_{x} \leq 0 \text { for } x>L_{2}, t>0 .
$$

Proof. By Lemma 6.1 and its proof, we know that

$$
u(t, x)=\lim _{\varepsilon \rightarrow 0} u_{\varepsilon}(t, x) \quad \text { and } \quad u_{x}(t, x)=\lim _{\varepsilon \rightarrow 0}\left(u_{\varepsilon}\right)_{x}(t, x)
$$

locally uniformly in $(0, \infty) \times \mathbb{R}^{1}$. Since Lemma 2.1 applies to $u_{\varepsilon}$, we have

$$
\left(u_{\varepsilon}\right)_{x}>0 \text { for } x<L_{1}, t>0 ; \quad\left(u_{\varepsilon}\right)_{x}<0 \text { for } x>L_{2}, t>0 .
$$

Letting $\varepsilon \rightarrow 0$, we obtain the required inequalities.

Lemma 6.3. Let $u$ and $u_{0}$ be as in Lemma 6.1, and suppose that $u_{0}$ is not identically 0 . Then (1.12) holds.

Proof. This follows by a careful examination of the proof of Proposition 1.8 Letting $g=f$, we find that there exists $M>0$ such that

$$
-M s \leq g(s) \leq M s \quad \text { for all } s \in[0, \sup u] .
$$

Therefore we still have (5.3) and 5.6. Since $\alpha_{n} \rightarrow 0$ and $g(u)$ is continuous in $[0,1)$, for all large $n$, the functions

$$
w \mapsto \frac{\varepsilon_{n}^{2}}{\alpha_{n}} g\left(\alpha_{n} w\right)
$$

have all the properties stated in the proof of Proposition 1.8 Hence 1.12 can be proved by the same argument.

Lemma 6.4. Let $J$ be an interval in $\mathbb{R}^{1}$ (finite or infinite) and let $\partial J$ denote its boundary. Let $u$ and $\tilde{u}$ satisfy the equation (1.1) in the weak sense on the domain $\left(t_{1}, t_{2}\right) \times J$ and suppose that $u \neq \tilde{u}$ on $\left(t_{1}, t_{2}\right) \times \partial J$. Then $\mathcal{Z}_{J}(u(t, \cdot)-\tilde{u}(t, \cdot))$ is nonincreasing in $t \in$ $\left(t_{1}, t_{2}\right)$. 
Proof. It suffices to show that, for any $t_{0} \in\left(t_{1}, t_{2}\right)$, the following holds:

$$
\mathcal{Z}_{J}\left(u\left(t_{0}, \cdot\right)-\tilde{u}\left(t_{0}, \cdot\right)\right) \geq \mathcal{Z}_{J}(u(t, \cdot)-\tilde{u}(t, \cdot)) \quad \text { for } t \in\left[t_{0}, t_{2}\right) .
$$

Let $f_{\varepsilon}$ be the Lipschitz approximation of $f$ given in the proof of Lemma 6.1, and let $u_{\varepsilon}, \tilde{u}_{\varepsilon}$ be the solution of (6.4) on the domain $\left[t_{0}, t_{2}\right) \times J$ with the following initial and the boundary conditions:

$$
\begin{array}{llll}
u_{\varepsilon}\left(t_{0}, x\right)=u\left(t_{0}, x\right) & (x \in \bar{J}), & u_{\varepsilon}=u & \text { on }\left(t_{0}, t_{2}\right) \times \partial J \\
\tilde{u}_{\varepsilon}\left(t_{0}, x\right)=\tilde{u}\left(t_{0}, x\right) & (x \in \bar{J}), & \tilde{u}_{\varepsilon}=\tilde{u} & \text { on }\left(t_{0}, t_{2}\right) \times \partial J .
\end{array}
$$

(Here, the boundary condition applies only if $\partial J \neq \emptyset$.)

Then by Lemma 2.3 , we have

$$
\mathcal{Z}_{J}\left(u\left(t_{0}, \cdot\right)-\tilde{u}\left(t_{0}, \cdot\right)\right)=\mathcal{Z}_{J}\left(u_{\varepsilon}\left(t_{0}, \cdot\right)-\tilde{u}_{\varepsilon}\left(t_{0}, \cdot\right)\right) \geq \mathcal{Z}_{J}\left(u_{\varepsilon}(t, \cdot)-\tilde{u}_{\varepsilon}(t, \cdot)\right)
$$

for $t \in\left[t_{0}, t_{2}\right)$. Arguing as in the proof of Lemma 6.1, we see that $u_{\varepsilon}$ and $\tilde{u}_{\varepsilon}$ converge to $u$ and $\tilde{u}$, respectively. Letting $\varepsilon \rightarrow 0$ in the above inequality and using (2.6) (which is easily seen to hold if $\mathcal{Z}$ is replaced by $\mathcal{Z}_{J}$ ), we obtain the desired inequality.

Lemma 6.5. Let $u$ be the solution of (1.1) given in Lemma 6.1 and $v$ a solution of (1.3). Suppose that $J=(a, b)$ is any finite open interval in $\mathbb{R}^{1}$ such that $0<v(x) \neq 1$ in $J, u(t, x)-v(x) \neq 0$ for $x \in\{a, b\}$ and for $t$ in some finite or infinite interval $\left(t_{1}, t_{2}\right) \subset[0, \infty)$. Then

$$
z(t):=\mathcal{Z}_{J}(u(t, \cdot)-v)
$$

is finite and nonincreasing in $t \in\left(t_{1}, t_{2}\right)$. Furthermore, if $u\left(t_{0}, x\right)-v(x)$ has a degenerate zero in $J$ for some $t_{0} \in\left(t_{1}, t_{2}\right)$, then $z\left(t_{0}-0\right)>z\left(t_{0}+0\right)$.

Proof. Fix $t_{0} \in\left(t_{1}, t_{2}\right)$. Suppose $u\left(t_{0}, x\right)-v(x)=0$ for some $x \in J$ and let $x_{0}$ be any such point. Then, since $u \neq v$ for $x=a, b$ and since both $u$ and $v$ are continuous and $v \neq 1$, we can find a small constant $\varepsilon>0$ and an interval $J_{x_{0}}$ with $x_{0} \in J_{x_{0}} \subset J$ such that

$$
\begin{gathered}
(u(t, x)-1)(v(x)-1)>0 \quad \text { for }(t, x) \in\left[t_{0}-\varepsilon, t_{0}+\varepsilon\right] \times J_{x_{0}}, \\
u(x, t) \neq v(x) \quad \text { for }(t, x) \in\left[t_{0}-\varepsilon, t_{0}+\varepsilon\right] \times \partial J_{x_{0}} .
\end{gathered}
$$

In this region $u(t, x)$ is away from the discontinuity point of $f$, and $u-v$ satisfies an equation of the form 2.5. Thus we can apply Lemma 2.3 to conclude that $x_{0}$ is an isolated zero of $u\left(t_{0}, x\right)-v(x)$. The fact that any zero of $u\left(t_{0}, x\right)-v(x)$ in $J$ is isolated and that the end points of $J$ are not zeros imply that there are only finitely many zeros of $u\left(t_{0}, x\right)-v(x)$ in $J$.

That $z(t)$ is nonincreasing follows from Lemma 6.4. Furthermore, if $u\left(t_{0}, x\right)-v(x)$ has a degenerate zero in $J$, say at $x=x_{0}$, then by Lemma 2.3 .

$$
\mathcal{Z}_{J_{x_{0}}}\left(u\left(t_{0}-0, \cdot\right)-v\right)>\mathcal{Z}_{J_{x_{0}}}\left(u\left(t_{0}+0, \cdot\right)-v\right),
$$

where $J_{x_{0}}$ is as above. On the other hand, by Lemma 6.4 .

$$
\mathcal{Z}_{J \backslash J_{x_{0}}}\left(u\left(t_{0}-0, \cdot\right)-v\right) \geq \mathcal{Z}_{J \backslash J_{x_{0}}}\left(u\left(t_{0}+0, \cdot\right)-v\right) .
$$

Hence $z\left(t_{0}-0\right)>z\left(t_{0}+0\right)$. The lemma is proved. 
We now examine the stationary solutions of 11.1 with $f$ satisfying $\left(\hat{\mathrm{f}}_{C}\right)$. We say that $v$ is a stationary solution of (1.1) if the (unique) weak solution $u$ of (1.1) with initial data $u_{0}=v$ satisfies $u \equiv v$. Let us first observe that any stationary solution $v$ satisfies

$$
v^{\prime \prime}+f(v-0) \geq 0, \quad v^{\prime \prime}+f(v+0) \leq 0
$$

in the weak sense. To see this, let $f_{\varepsilon}(\geq f)$ denote the Lipschitz approximation of $f$ defined in the proof of Lemma 6.1. Then $v$ is a lower solution of the approximate problem, that is, $v^{\prime \prime}+f_{\varepsilon}(v) \geq 0$ in the weak sense. Letting $\varepsilon \rightarrow 0$, we obtain the first inequality of 6.5). The second inequality can be shown similarly by using a Lipschitz approximation of $f$ from below.

From 6.5 we see that $v \equiv 1$ is a stationary solution even though it does not satisfy (1.3) when $f(1-0)>0>f(1+0)$. It is also clear that any stationary solution $v$ satisfies 1.3 at every $x \in \mathbb{R}^{1}$ where $v(x) \neq 1$. In view of this, one can show that nonnegative bounded stationary solutions of 11.1 consist of constant functions $v \equiv c$ with $c \in\left[0, \theta_{0}\right] \cup\{1\}$ as in the case $\left(\mathrm{f}_{C}\right)$.

Next we consider solutions of (1.3) satisfying

$$
v\left(x_{0}\right)=\alpha \in(0,1), \quad v^{\prime}\left(x_{0}\right)=0
$$

for some $x_{0} \in \mathbb{R}^{1}$. Since $f$ satisfies $\left(\hat{\mathrm{f}}_{C}\right)$, it is easily seen that $v$ is either a constant solution (when $\left.\alpha \in\left(0, \theta_{0}\right]\right)$, or a solution with compact positive support (when $\alpha \in\left(\theta_{0}, 1\right)$ ). Thus in all the possible cases, we have

$$
v(x)<1 \quad \text { whenever it is defined. }
$$

In what follows, the symbol $\operatorname{spt}^{+}(v)$ will denote the positive support of a function $v$ as defined in Subsection 2.5 .

Lemma 6.6. Let $u$ be the solution of (1.1) given in Lemma 6.1, and $v$ a solution of (1.3) satisfying 6.6. Then

$$
\mathcal{Z}_{I}(u(t, \cdot)-v)<\infty \text { for } t>0,
$$

and it is nonincreasing in $t$, where $I=\operatorname{spt}^{+}(v)$.

Proof. The proof is the same as that of Lemma 2.9. Here we use Lemma 6.5 instead of Lemma 2.3.

We are now ready to prove the convergence theorem.

Theorem 6.7 (Convergence for the discontinuous combustion case). Assume $\left(\hat{\mathrm{f}}_{C}\right)$ and let $u$ be the weak solution of (1.1). Then $\lim _{t \rightarrow \infty} u(t, x)$ exists in the topology of $L_{\mathrm{loc}}^{\infty}\left(\mathbb{R}^{1}\right)$, and the limit is a constant $c \in\left\{0, \theta_{0}, 1\right\}$.

Proof. We first show that $\omega(u)$ consists of stationary solutions. This is done by slightly modifying the proof of Lemma 3.4. Let $w$ be any element of $\omega(u)$. By Lemma 6.1. we have $0 \leq w \leq 1$. If $w \equiv 1$, then the proof is over, so we suppose that $w \neq \equiv 1$. Then there exists a sequence $x_{n} \in \mathbb{R}^{1}(n=1,2, \ldots)$ such that

$$
w\left(x_{n}\right) \rightarrow \beta:=\inf _{x \in \mathbb{R}^{1}} w(x), \quad w^{\prime}\left(x_{n}\right) \rightarrow 0 \quad \text { as } n \rightarrow \infty .
$$


Denote by $v_{n}(x)(n=1,2, \ldots)$ a sequence of solutions of 1.3 satisfying

$$
v_{n}\left(x_{n}\right)=w\left(x_{n}\right), \quad v_{n}^{\prime}\left(x_{n}\right)=w^{\prime}\left(x_{n}\right) .
$$

Here the domain of definition of $v_{n}$ is taken to be the maximal interval that contains $x_{n}$ and on which $0 \leq v_{n}<1$. Now define

$$
H(v, p):=\frac{1}{2} p^{2}+F(v), \quad F(v):=\int_{0}^{v} f(s) d s .
$$

Then $H\left(v_{n}(x), v_{n}^{\prime}(x)\right)$ is independent of $x$ and $H\left(v_{n}\left(x_{n}\right), v_{n}^{\prime}\left(x_{n}\right)\right) \rightarrow H(\beta, 0)=F(\beta)$ as $n \rightarrow \infty$. Since $F(v)=0$ for $0 \leq v \leq \theta_{0}$ and $F(v)$ is strictly increasing in $\theta_{0}<v<1$, we have $F(\beta)<F(\gamma) \leq H(\gamma, p)$, where $\gamma$ is any point satisfying $\max \left\{\beta, \theta_{0}\right\}<\gamma<1$. Fix such a $\gamma$. Then, for sufficiently large $n$,

$$
H\left(v_{n}, v_{n}^{\prime}\right)<H(\gamma, p) \quad(\forall p \in \mathbb{R}),
$$

which implies that $v_{n}(x)$ cannot attain the value $\gamma$; therefore $\max v_{n}<\gamma<1$.

Hence $v_{n}$ is either a constant solution of 1.3 or a solution with compact positive support and $\max v_{n}<1$. Thus Lemma 6.6 applies for such $v=v_{n}$. Arguing as in the proof of Lemma 3.4 (while using Lemma 6.6 instead of Lemma 2.9. and Lemma 6.5 instead of Lemma 2.3, we find that $w$ coincides with such $v_{n}$. Therefore $w$ is either a constant or a solution of 1.3 with compact positive support. But the latter is impossible since $w$ is smooth and nonnegative. Hence $w$ is a constant function with value in the interval $\left[0, \theta_{0}\right]$, provided that $w \neq \equiv 1$.

It remains to show that $\omega(u)$ is a singleton, and that its element is either $0, \theta_{0}$ or 1 . Since the proof of Lemma 3.5 is valid once we replace Lemma 2.1 by Lemma 6.2, we have $\omega(u) \subset\left\{0, \theta_{0}, 1\right\}$. The connectedness of $\omega(u)$ then implies that $\omega(u)$ is a singleton. This completes the proof of the theorem.

Theorem 6.8 (Threshold for the discontinuous combustion case). The conclusions in Theorem 1.4 remain valid under the assumption $\left(\hat{\mathrm{f}}_{C}\right)$.

Proof. We first note that the usual comparison principle continues to hold. Indeed, this follows from the same argument as in the proof of Lemma 6.1 for uniqueness. The theorem can then be shown by repeating the proof of Theorem 1.4 in Subsection 4.2 with only minor modifications. First, the proofs of Lemmas 4.7, 4.8 and 4.9 go without change. The proof of Lemma 4.11 carries over when Lemma 6.3 is used instead of Proposition 1.8 (which was used to derive (4.10)), and when we notice that only the inequality $\left|f\left(u_{i}\right)\right| \leq M u_{i}$ was needed rather than the Lipschitz continuity of $f$. The conclusion of Lemma 4.10 is then proved in the same way except that Theorem 6.7 is used instead of Theorem 1.1

\subsection{The bistable case}

In this subsection we consider the case where $f$ satisfies $\left(\mathrm{f}_{B}\right)$ with a jumping discontinuity at $u=1$. As we have mentioned in the previous subsection, discontinuity of the type 
$f(1-0)=0>f(1+0)$ can be treated precisely the same way as the type $f(1-0)>$ $0 \geq f(1+0)$, so we focus on the latter. Thus we assume:

$$
\left(\hat{\mathrm{f}}_{B}\right)\left\{\begin{array}{l}
f \text { is Lipschitz in }[0,1) \text { and locally Lipschitz in }[1, \infty) \\
f \text { satisfies } 1.4,(1.5 \text { and } 6.1 .
\end{array}\right.
$$

Our main results in this subsection are the following:

Theorem 6.9 (Convergence for the discontinuous bistable case). Assume $\left(\hat{\mathrm{f}}_{B}\right)$ and let $u$ be the weak solution of $(1.1)$. Then $\lim _{t \rightarrow \infty} u(t, x)$ exists in the topology of $L_{\mathrm{loc}}^{\infty}\left(\mathbb{R}^{1}\right)$, and the limit is either 0,1 , or a symmetrically decreasing stationary solution $U(x-a)$ for some $a \in \mathbb{R}$, where $U$ is as in 1.8 .

Theorem 6.10 (Threshold for the discontinuous bistable case). The conclusions in Theorem 1.3 remain valid under the assumption $\left(\hat{\mathrm{f}}_{B}\right)$.

Proof of Theorem 6.9. We first show that $\omega(u)$ consists only of stationary solutions. The proof is exactly the same as the corresponding part of the proof of Theorem 6.7 except that the function $v_{n}$ is now either the constant solution $\theta_{0}$, a periodic solution satisfying $0<v_{n}<1$, a symmetrically decreasing solution with max $v_{n}<1$, or a solution with compact positive support and $\max v_{n}<1$. In all these cases, Lemma 6.6 applies, so one can just repeat the proof of Theorem 6.7 to show that $w$ coincides with $v_{n}$ and that $v_{n}$ is either 0 or a positive stationary solution.

It remains to show that $\omega(u)$ is a singleton. We first observe that Lemma 3.1 still holds because of Lemma 6.2. Therefore any element of $\omega(u)$ is either a symmetrically decreasing solution of the form $U(x-a)$, or one of the constant solutions $0, \theta_{0}, 1$. But the constant $\theta_{0}$ cannot belong to $\omega(u)$ by virtue of Lemma 3.3, since $\theta_{0}$ intersects periodic solutions infinitely many times. (Note that Lemma 3.3 remains valid by virtue of Lemma 6.6.) Thus

$$
\omega(u) \subset\{0,1\} \cup\{U(x-a): a \in \mathbb{R}\} .
$$

If $\omega(u)$ contains 1 , then by the connectedness of $\omega(u)$ we have $\omega(u)=\{1\}$, and the proof is over. Next suppose $\omega(u) \subset\{0\} \cup\{U(x-a): a \in \mathbb{R}\}$. Then by Lemma 6.2, we have $\max _{x} u(t, x)<1$ for all sufficiently large $t$; hence $u$ is a classical solution of (1.1) for large $t$. Consequently, we can apply Lemma 2.8 to derive Lemma 3.2. This implies that $\omega(u)$ is a singleton, and the proof of the theorem is complete.

Proof of Theorem 6.10. The proof is identical to that of Theorem 1.3 except that Lemma 4.5 is derived from Lemma 6.3 rather than from Proposition 1.8 , The details are omitted.

Acknowledgments. The authors are grateful to Professor Haim Brezis for drawing their attention to the importance of the case of discontinuous nonlinearity and sending us [BKS]. This research was initiated when the first author was visiting the second author at the University of Tokyo under a Bilateral Exchange Program sponsored by the Japan Society for the Promotion of Science and the Australian Academy of Science. He would like to thank the University of Tokyo for providing excellent research environment and warm hospitality, and wishes to acknowledge the support of the Bilateral Exchange Program. 


\section{References}

[AC] Allen, S., Cahn, J.: A microscopic theory for antiphase boundary motion and its application to antiphase domain coarsening. Acta Metallica 27, 1084-1095 (1979)

[An] Angenent, S. B.: The zero set of a solution of a parabolic equation. J. Reine Angew. Math. 390, 79-96 (1988) Zbl 0644.35050 MR 0953678

[AW1] Aronson, D. G., Weinberger, H. F.: Nonlinear diffusion in population genetics, combustion, and nerve pulse propagation. In: Partial Differential Equations and Related Topics, Lecture Notes in Math. 446, Springer, Berlin, 5-49 (1975) Zbl 0325.35050 MR 0427837

[AW2] Aronson, D. G., Weinberger, H. F.: Multidimensional nonlinear diffusion arising in population genetics. Adv. Math. 30, 33-76 (1978) Zbl 0407.92014 MR 0511740

[BN] Berestycki, H., Nirenberg, L.: Travelling fronts in cylinders. Ann. Inst. H. Poincaré Anal. Non Linéaire 9, 497-573 (1992) Zbl 0799.35073 MR 1191008

[BKS] Brezis, H., Kamin, S., Sivashinsky, G.: Initiation of subsonic detonation. Asymptotic Anal. 24, 73-90 (2000) Zbl 0989.35029 MR 1792726

[BJP] Busca, J., Jendoubi, M. A., Poláčik, P.: Convergence to equilibrium for semilinear parabolic problems in $\mathbb{R}^{N}$. Comm. Partial Differential Equations 27, 1793-1814 (2002) Zbl 1021.35013 MR 1941658

[C1] Chen, X.-Y.: Uniqueness of the $\omega$ limit point of solutions of a semilinear heat equation on the circle. Proc. Japan Acad. Ser. A 62, 335-337 (1986) Zbl 0641.35028 MR 0888140

[CM] Chen, X.-Y., Matano, H.: Convergence, asymptotic periodicity, and finite-point blowup in one-dimensional semilinear heat equations. J. Differential Equations 78, 160-190 (1989) Zbl 0692.35013 MR 0986159

[FP] Feireisl, E., Poláčik, P.: Structure of periodic solutions and asymptotic behavior for timeperiodic reaction-diffusion equations on $\mathbb{R}$. Adv. Differential Equations 5, 583-622 (2000) Zbl 0987.35079 MR 1750112

[FM] Fife, P. C., McLeod, J. B.: The approach of solutions of nonlinear diffusion equations to travelling front solutions. Arch. Ration. Mech. Anal. 65, 335-361 (1977) Zbl 0361.35035 MR 0442480

[F] Fisher, R. A.: The advance of advantageous genes. Ann. of Eugenics 7, 355-369 (1937) JFM 63.1111.04

[J] Jones, C. K. R. T.: Spherically symmetric solutions of a reaction-diffusion equation. J. Differential Equations 49, 142-169 (1983) Zbl 0523.35059 MR 0704268

[K] Kanel', Ya. I.: Stabilization of the solutions of the equations of combustion theory with finite initial functions. Mat. Sb. (N.S.) 65 (107), 398-413 (1964) (in Russian) MR 0177209

[KPP] Kolmogorov, A. N., Petrovskiŭ, I. G., Piskunov, N. S.: A study of the equation of diffusion with increase in the quantity of matter, and its applications to a biological problem. Bull. Moskov. Gos. Univ. Mat. Mekh. 1, 1-25 (1937)

[LSU] Ladyzhenskaya, O. A., Solonnikov, V. A., Ural'tseva, N. N.: Linear and Quasilinear Equations of Parabolic Type. Amer. Math. Soc. (1968) Zbl 0174.15403 MR 0241822

[L] Lieberman, G..: Second Order Parabolic Differential Equations, World Sci., Singapore (1996) Zbl 0884.35001 MR 1465184

[M] Matano, H.: Convergence of solutions of one-dimensional semilinear parabolic equations. J. Math. Kyoto Univ. 18, 221-227 (1978) Zbl 0387.35008 MR 0501842

[NAY] Nagumo, J., Arimoto, S., Yoshizawa, S.: An active pulse transmission line simulating nerve axon. Proc. Inst. Radio Engrg. 50, 2061-2070 (1962) 
[ZFK] Zel'dovich, Ya. B., Frank-Kamenetski1, D. A.: A theory of thermal propagation of flame. Acta Physiochimica USSR 9, 341-350 (1938)

[Ze] Zelenyak, T. I.: Stabilization of solutions of boundary value problems for a secondorder parabolic equation with one space variable. Differentsial'nye Uravneniya 4, 17-22 (1968) (in Russian) Zbl 0232.35053

[Z] Zlatoš, A.: Sharp transition between extinction and propagation of reaction. J. Amer. Math. Soc. 19, 251-263 (2006) Zbl 1081.35011 MR 2169048 